\title{
Transverse-momentum resummation for top-quark pair production at the LHC
}

\author{
Stefano Catani, ${ }^{a}$ Massimiliano Grazzini ${ }^{b}$ and Hayk Sargsyan ${ }^{b}$ \\ ${ }^{a}$ INFN, Sezione di Firenze and Dipartimento di Fisica e Astronomia, Università di Firenze, \\ Via Sansone 1, I-50019 Sesto Fiorentino, Florence, Italy \\ ${ }^{b}$ Physik-Institut, Universität Zürich, \\ Winterthurerstrasse 190, CH-8057 Zurich, Switzerland \\ E-mail: catani@fi.infn.it, grazzini@physik.uzh.ch, shayk@physik.uzh.ch
}

ABSTRACT: We consider transverse-momentum resummation for top-quark pair production in hadron collisions. At small transverse momenta of the top-quark pair, the logarithmically-enhanced QCD contributions are resummed to all orders up to next-toleading logarithmic accuracy. At intermediate and large values of transverse momenta, the resummation is consistently combined with the complete result at fixed perturbative order. We present numerical results for the transverse-momentum distribution of top-quark pairs at LHC energies. We perform a detailed study of the scale dependence of the results to estimate their perturbative uncertainty. We comment on the comparison with ATLAS and CMS data.

KeYwords: Perturbative QCD, Resummation

ArXiv ePrint: 1806.01601 


\section{Contents}

1 Introduction 1

2 Transverse-momentum resummation 3

$\begin{array}{llr}3 & \text { Results } & 7\end{array}$

3.1 Fixed-order results 8

$\begin{array}{lll}3.2 & \text { Resummed results } & 13\end{array}$

$\begin{array}{lll}4 & \text { Summary } & 24\end{array}$

\section{Introduction}

The top quark $(t)[1]$ is the heaviest known elementary particle. Since its mass is close to the scale of electroweak symmetry breaking, the top quark is crucial to the hierarchy problem. Within the Standard Model (SM) the main source of top-quark events in highenergy hadron collisions is the production of top-quark pairs. The study of top-quark pair $(t \bar{t})$ production at hadron colliders can shed light on the nature of the electroweaksymmetry breaking. Moreover, such a study also gives relevant information on the SM backgrounds of many new-physics models.

The theoretical efforts for obtaining accurate predictions for $t \bar{t}$ production at hadron colliders started three decades ago with the calculation of the next-to-leading order (NLO) QCD corrections to the total cross section [2-5] and kinematical distributions [6] for this production process. The calculation of the next-to-next-to-leading order (NNLO) QCD corrections to the $t \bar{t}$ total cross section was completed [7-10] in recent years. Besides the total cross section, NNLO predictions for differential cross sections and more general kinematical distributions are now available [11-14] and are of great importance for precision studies.

This paper is devoted to the transverse-momentum spectrum of the top-quark pair. This observable has been measured by the ATLAS and CMS experiments at the LHC in proton-proton collisions at the centre-of-mass energies $\sqrt{s}=7 \mathrm{TeV}[15-17]$ and $8 \mathrm{TeV}$ [1720]. First results at $\sqrt{s}=13 \mathrm{TeV}[21-24]$ started to appear recently.

The bulk of the cross section is produced in the kinematical region where the transverse momentum $q_{T}$ of the top-quark pair is smaller than the mass $m_{t}$ of the top quark. The $q_{T}$ differential cross section $d \sigma / d q_{T}$ of the top-quark pair is computable in QCD perturbation theory [6, 25-28]. In the small- $q_{T}$ region $\left(q_{T} \ll m_{t}\right)$, the perturbative expansion is badly behaved, since the size of the perturbative coefficients is enhanced by powers of $\ln q_{T}$. A reliable theoretical calculation of the detailed $q_{T}$ shape of $d \sigma / d q_{T}$ requires the all-order resummation of these logarithmically-enhanced terms. 
This type of perturbative behaviour at small $q_{T}$ is well known [29-32] from the hadroproduction process of a high-mass lepton pair through the Drell-Yan (DY) mechanism. In the case of the DY process the all-order resummation of the $\ln q_{T}$ terms is fully understood [29-33]. At the level of leading-logarithmic (LL) contributions, the extension of resummation from the DY process to the heavy-quark process is relatively straightforward $[34,35]$. However, beyond the LL level the structure of $\ln q_{T}$ terms for the heavy-quark process is definitely different from that of the DY process. Indeed, transverse-momentum resummation for the DY process, and, more generally, for the production of colourless high-mass systems, has an all-order universal (process-independent) structure. This universality structure eventually originates from the fact that the transverse momentum of the colourless system is produced by (soft and collinear) QCD radiation from the initial-state colliding partons. The heavy-quark production process definitely belongs to a different class of processes, since the produced final-state heavy quarks carry colour charge and, therefore, they act as additional source of QCD radiation. The transverse momentum of the heavy-quark pair depends on initial-state radiation, on final-state radiation and on quantum (and colour flow) interferences between radiation from the initial and final states. These physical differences lead to conceptual and technical complications in the theoretical formulation of transverse-momentum resummation for heavy-quark production.

The all-order $q_{T}$ resummation for the heavy-quark production process was discussed in refs. [36-38]. The analysis of refs. [36, 37] is based on Soft Collinear Effective Theory (SCET), and it is limited to the treatment of the $q_{T}$ cross section averaged over the azimuthal angles of the produced heavy quarks. Corresponding quantitative results for $t \bar{t}$ production at the LHC were obtained in refs. [36, 37] by following and extending the implementation formalism of ref. [39]. An independent formulation of transverse-momentum resummation for heavy-quark pairs, including the complete treatment of azimuthal correlations is presented in ref. [38]. A main difference between the production of heavy quarks and of colourless systems is the appearance of a new resummation factor that is due to softparton radiation at wide angles with respect to the direction of the colliding hadrons. This additional resummation factor embodies the effect of soft radiation from the heavy-quark final state and from initial-state and final-state interferences. This factor is controlled by a soft anomalous dimension that depends on the angular (rapidity) distribution of the produced heavy quark and antiquark and on the colour configuration of their underlying production mechanism.

In this paper we present quantitative results for the transverse-momentum spectrum of a top-quark pair at the LHC. The results are obtained by using the all-order resummation formalism of ref. [38], which is directly implemented in impact parameter space [40]. We perform the resummation up to the next-to-leading logarithmic (NLL) level, by also explicitly including all (logarithmic and non logarithmic) contributions up to NLO in the perturbative QCD expansion. We study the perturbative uncertainty of our results and compare them to the available LHC data at $\sqrt{s}=8 \mathrm{TeV}[17,18]$. We also study the quantitative impact of the soft wide-angle factor that is a distinctive feature of heavy-quark production. Finally we present a comparison of our resummed results for the $t \bar{t}$ spectrum with those obtained by using a Monte Carlo generator that interfaces the NLO calculation to the PYTHIA parton shower [41] by using the POWHEG BOX implementation [42]. 
The quantitative results presented in this paper are limited to the $q_{T}$ cross section integrated over the azimuthal angles at the $t \bar{t}$ pair. Corresponding results for azimuthal correlations of the top-quark pair have been presented in ref. [43]. Those results are included in a more general (process-independent) discussion [43] of highly non-trivial features related to azimuthal correlation effects in QCD.

The paper is organized as follows. In section 2 we briefly recall the theoretical framework and the resummation formalism that we use to carry out our calculation. In section 3 we present our numerical results and comparisons with LHC data. In section 3.1 we consider fixed-order calculations at NLO and NNLO, and then in section 3.2 we present our resummed predictions. Our results are summarized in section 4 .

\section{Transverse-momentum resummation}

The resummation formalism that we use in this paper is discussed in detail in ref. [38]. The formalism can be applied to a generic pair of heavy quarks that is produced in hadronhadron collisions. In this section we briefly recall the main points of the formalism, by focusing on the specific case of the transverse-momentum spectrum of a $t \bar{t}$ pair.

We consider the inclusive hard-scattering process $h_{1}+h_{2} \rightarrow t \bar{t}+X$, where the two colliding hadrons $h_{1}$ and $h_{2}$ with centre-of-mass energy $\sqrt{s}$ produce the $t \bar{t}$ pair, and $X$ denotes the accompanying final-state radiation. Although we are mostly interested in the single-differential cross section $d \sigma / d q_{T}$, in our presentation we consider the tripledifferential cross section with respect to $q_{T}$, the invariant mass $M$ of the $t \bar{t}$ pair and the scattering angle of the quark or antiquark. Indeed, the use of the angular dependent cross section clarifies how resummation can be systematically organized in exponential form (see eqs. (2.4) and (2.5) and the ensuing discussion in this section). The differential cross section at fixed values of $q_{T}, M$ and of the polar angle $\theta$ of the top quark in the Collins-Soper rest frame $^{1}[44]$ of the $t \bar{t}$ pair can be written as

$$
\begin{aligned}
\frac{d \sigma}{d q_{T}^{2} d M^{2} d \cos \theta}\left(q_{T}, M, s, \theta\right)= & \sum_{a, b} \int_{0}^{1} d x_{1} \int_{0}^{1} d x_{2} f_{a / h_{1}}\left(x_{1}, \mu_{F}^{2}\right) f_{b / h_{2}}\left(x_{2}, \mu_{F}^{2}\right) \\
& \times \frac{d \hat{\sigma}_{a b}}{d q_{T}^{2} d M^{2} d \cos \theta}\left(q_{T}, M, \hat{s}, \theta ; \alpha_{\mathrm{S}}\left(\mu_{R}^{2}\right), \mu_{R}^{2}, \mu_{F}^{2}\right)
\end{aligned}
$$

where $a, b$ denotes the parton indices $(a=q, \bar{q}, g), f_{a / h}\left(x, \mu_{F}^{2}\right)$ are the parton distribution functions (PDFs) of the colliding hadron $h$ at the factorization scale $\mu_{F}, d \hat{\sigma}_{a b}$ is the partonic differential cross section for the partonic process $a+b \rightarrow t \bar{t}+X, \hat{s}=x_{1} x_{2} s$ is the square of the partonic centre-of-mass energy and $\alpha_{\mathrm{S}}\left(\mu_{R}^{2}\right)$ is the QCD coupling evaluated at the renormalization scale $\mu_{R}$. We use the $\overline{\mathrm{MS}}$ renormalization scheme for the QCD coupling and the $\overline{\mathrm{MS}}$ factorization scheme for the PDFs. The cross sections $d \sigma$ and $d \hat{\sigma}$ obviously depend on the mass $m_{t}$ of the top quark, but the $m_{t}$ dependence is not explicitly denoted in all the formulae of this section.

\footnotetext{
${ }^{1}$ The polar angle in other rest frames of the $t \bar{t}$ pair can equivalently be used (see appendix A in ref. [45]).
} 
The resummation is performed at the level of the partonic cross section, which is first decomposed as [38]

$$
d \hat{\sigma}=d \hat{\sigma}^{(\operatorname{sing})}+d \hat{\sigma}^{(\mathrm{reg})}
$$

where, order-by-order in perturbation theory, the component $d \hat{\sigma}^{(\text {sing) }}$ embodies all the singular terms in the $q_{T} \rightarrow 0$ limit, whereas $d \hat{\sigma}^{(\mathrm{reg})}$ includes the remaining non-singular terms. At the $n$-th order in the expansion in powers of $\alpha_{\mathrm{S}}$ we thus have $d \hat{\sigma}^{(\mathrm{reg})} / d \hat{\sigma}^{(\mathrm{sing})}=\mathcal{O}\left(q_{T} / M\right)$ (modulo logarithmic corrections) and $d \hat{\sigma}^{(\mathrm{reg})}$ represents a power suppressed correction (by at least one power of $\left.q_{T} / M\right)$ to $d \hat{\sigma}^{(\text {sing })}$ as $q_{T} \rightarrow 0$.

In our resummation treatment the 'singular' component $d \hat{\sigma}^{(\mathrm{reg})}$, which contains all the singular logarithmically-enhanced contributions at small $q_{T}$, is evaluated by resumming these contributions to all order in $\alpha_{\mathrm{S}}$. The 'regular' component $d \hat{\sigma}^{(\mathrm{reg})}$ is instead evaluated at a specified fixed-order accuracy.

The resummation procedure of the logarithmically-enhanced terms in $d \hat{\sigma}^{(\operatorname{sing})}$ is carried out in the impact parameter space. The impact parameter $\mathbf{b}$ is the conjugate variable to $\mathbf{q}_{T}$ through a Fourier transformation. The small- $q_{T}$ region $\left(q_{T} \ll M\right)$ corresponds to the large- $b$ region $(M b \gg 1)$ and the logarithmic terms $\ln \left(q_{T} / M\right)$ become large logarithmic contributions $\ln \left(M^{2} b^{2}\right)$ in $b$ space. The resummed component of the partonic cross section is then obtained by performing the inverse Fourier transformation with respect to the impact parameter $b$. For the azimuthally-integrated $[38,43]$ transverse-momentum partonic cross section the Fourier transformation turns into a Bessel transformation, and the singular component of the partonic cross section in the right-hand side of eq. (2.1) can be written as

$$
\frac{d \hat{\sigma}_{a_{1} a_{2}}^{\text {(sing) }}}{d q_{T}^{2} d M^{2} d \cos \theta}=\alpha_{\mathrm{S}}^{2} \sum_{c=q, \bar{q}, g} \frac{d \hat{\sigma}_{c \bar{c}}^{(0)}(M, \hat{s}, \theta)}{d M^{2} d \cos \theta} \int_{0}^{\infty} d b \frac{b}{2} J_{0}\left(b q_{T}\right) \mathcal{W}_{c \bar{c} \leftarrow a_{1} a_{2}}\left(b, M, \hat{s}, \theta ; \alpha_{\mathrm{S}}, \mu_{R}^{2}, \mu_{F}^{2}\right)
$$

where $J_{0}(x)$ is the 0th-order Bessel function, and $\alpha_{\mathrm{S}}^{2} d \hat{\sigma}_{c \bar{c}}^{(0)}$ is the leading-order (LO) differential cross section for the partonic process $c+\bar{c} \rightarrow t \bar{t}$. As explicitly denoted by the sum over $c$ in eq. (2.3), the LO process only involves the two partonic channels of quark-antiquark annihilation $(c \bar{c}=q \bar{q}, \bar{q} q)$ and gluon fusion $(c \bar{c}=g g)$. The resummation of high-order radiative corrections is embodied in the $b$-space resummed factor $\mathcal{W}_{c \bar{c} \leftarrow a_{1} a_{2}}$.

The all-order resummation structure of $\mathcal{W}_{c \bar{c} \leftarrow a_{1} a_{2}}$ can be organized in exponential form [38]. This structure is better expressed by defining [40] the Mellin $N$-moments $\mathcal{W}_{N}(M)$ of $\mathcal{W}(M, \hat{s})$ with respect to the variable $z=M^{2} / \hat{s}$ at fixed $M$. For the sake of simplicity, in the subsequent presentation we omit the explicit dependence on the parton indices and also the dependence on colour indices (we postpone some comments on the colour structure and colour correlations). The $N$-moment $\mathcal{W}_{N}$ of the resummed factor $\mathcal{W}$ in eq. (2.3) has the form

$$
\mathcal{W}_{N}\left(b, M, \theta ; \alpha_{\mathrm{S}}, \mu_{R}^{2}, \mu_{F}^{2}\right)=\mathcal{H}_{N}\left(M, \theta ; \alpha_{\mathrm{S}}, \mu_{R}^{2}, \mu_{F}^{2}, Q\right) \exp \left\{\mathcal{G}_{N}\left(\theta, \alpha_{\mathrm{S}}, \tilde{L} ; M / \mu_{R}, M / Q\right)\right\}
$$


where the exponent $\mathcal{G}$ can be expanded as

$$
\begin{aligned}
\left.\mathcal{G}_{N}\left(\theta, \alpha_{\mathrm{S}}, \tilde{L} ; M / \mu_{R}, M / Q\right)\right\}= & \tilde{L} g^{(1)}\left(\alpha_{\mathrm{S}} \tilde{L}\right)+g_{N}^{(2)}\left(\theta, \alpha_{\mathrm{S}} \tilde{L} ; M / \mu_{R}, M / Q\right) \\
& +\sum_{k=3}^{\infty} \alpha_{\mathrm{S}}^{k-2} g_{N}^{(k)}\left(\theta, \alpha_{\mathrm{S}} \tilde{L} ; M / \mu_{R}, M / Q\right)
\end{aligned}
$$

The dependence of $\mathcal{G}$ on the impact parameter $b$ is encoded in the logarithmic expansion parameter

$$
\tilde{L} \equiv \ln \left(Q^{2} b^{2} / b_{0}^{2}+1\right),
$$

where $b_{0}=2 e^{-\gamma_{E}}\left(\gamma_{E}=0.5772 \ldots\right.$ is the Euler number $)$ and the auxiliary scale $Q(Q \sim M)$ is named resummation scale [46]. Note that the right-hand side of eq. (2.5) is not a customary order-by-order expansion in $\alpha_{\mathrm{S}}$, but rather an expansion in $\alpha_{\mathrm{S}}$ at fixed $\alpha_{\mathrm{S}} \tilde{L}$. Such expansion consistently resums classes of large logarithmic terms $\left(\tilde{L} \gg 1, \alpha_{\mathrm{S}} \tilde{L} \sim\right.$ $\mathcal{O}(1))$. The function $g^{(1)}$ controls the LL contributions $\alpha_{\mathrm{S}}^{n} \tilde{L}^{n+1}$ to $\mathcal{G}_{N}, g_{N}^{(2)}$ controls the NLL contributions $\alpha_{\mathrm{S}}^{n} \tilde{L}^{n}$, and so forth. The resummation scale $Q$ is introduced [46] to parametrize the arbitrariness in the truncation of the logarithmic expansion in eq. (2.5). Changing the value of $Q$ produces effects on $g_{N}^{(k)}(k \geq 2)$ that are parametrically of the same order as those due to $\alpha_{S} g_{N}^{(k+1)}$. Therefore, variations of $Q$ around the value $Q=M$ at a given logarithmic accuracy can be used to estimate uncertainties due to neglected subdominant classes of logarithmic contributions. The function $\mathcal{H}$ in eq. (2.4) does not depend on the impact parameter $b$ and, therefore, it can be expanded in powers of $\alpha_{\mathrm{S}}\left(\mu_{R}^{2}\right)$ :

$$
\mathcal{H}_{N}=\mathcal{H}_{N}^{(0)}+\left(\frac{\alpha_{\mathrm{S}}}{\pi}\right) \mathcal{H}_{N}^{(1)}+\left(\frac{\alpha_{\mathrm{S}}}{\pi}\right)^{2} \mathcal{H}_{N}^{(2)}+\mathcal{O}\left(\alpha_{\mathrm{S}}^{3}\right),
$$

where each perturbative term $\mathcal{H}_{N}^{(k)}$ depends on $M, \theta$ and the auxiliary scales $\mu_{R}, \mu_{F}, Q$.

Since the factor $\mathcal{H}_{N}$ in eq. (2.4) does not contain logarithmically-enhanced contributions, we treat it by truncation of the perturbative expansion in eq. (2.7) at a given fixed-order accuracy, analogously to the treatment of the regular component $d \hat{\sigma}^{(\mathrm{reg})}$ of the cross section in eq. (2.2).

The computation of $d \hat{\sigma}^{(\mathrm{sing})}$ and $d \hat{\sigma}^{(\mathrm{reg})}$ at a given logarithmic and fixed-order accuracy, respectively, defines a systematic expansion [40] of eq. (2.2). This expansion can be used to obtain predictions that contain the full information of the perturbative calculation up to a given fixed order plus resummation of the logarithmically-enhanced contributions at small $q_{T}$. The various orders of this expansion are denoted by LL, NLL+NLO, NNLL+NNLO and so forth, where the first label (LL, NLL, NNLL,...) denotes the logarithmic accuracy, while the second label $(\mathrm{NLO}, \mathrm{NNLO}, \ldots$ ) refers to the corresponding perturbative order of the total cross section. In particular, the NLL+NLO expansion of eq. (2.2) is obtained by including the functions $g^{(1)}$ and $g_{N}^{(2)}$ and the coefficients $\mathcal{H}^{(1)}$ in the resummed component, and by computing the finite component $d \hat{\sigma}^{(\mathrm{reg})}$ at $\mathcal{O}\left(\alpha_{\mathrm{S}}^{3}\right)$.

We remark that the NLL+NLO (NNLL+NNLO) result includes the full NLO (NNLO) perturbative contribution in the small- $q_{T}$ region. Considering a generic upper limit value $q_{T \max }$, the integral over the range $0 \leq q_{T} \leq q_{T \max }$ of the $q_{T}$ differential cross section at the NLL+NLO (NNLL+NNLO) level includes the complete perturbative terms up to NLO 
(NNLO). In particular, since the logarithmic variable $\tilde{L}$ in eq. (2.6) vanishes at $b=0$, the NLO (NNLO) total ( $q_{T}$ integrated) cross section $d \sigma / d M^{2} d \cos \theta$ is exactly recovered $[40,46]$ upon integration over $q_{T}$ of the NLL+NLO (NNLL+NNLO) result. In the case of heavyquark production, the coefficient $\mathcal{H}^{(2)}$ is still unknown: this prevents us from performing calculations at NNLL+NNLO accuracy. In the following sections of this paper we thus limit ourselves to presenting resummed results at NLL+NLO accuracy.

In eqs. (2.1)-(2.7) and accompanying comments we have briefly summarized the main results of ref. [38] on $q_{T}$ resummation for $t \bar{t}$ production. In this presentation we have used a notation and a style that closely follow analogous results [40] for production processes of colourless high-mass systems (e.g., the DY process or Higgs boson production). As discussed in ref. [38] and recalled in section 1, there are important differences between $t \bar{t}$ production and the production of colourless systems. In the following we highlight more explicitly these differences.

The LL function $g^{(1)}$ in eq. (2.5) is completely analogous to the corresponding function for colourless production. More precisely, taking into account the dependence on the parton indices (which has been neglected in the notation of eqs. (2.4) and (2.5)), $g^{(1)}$ depends on the flavour of the partons (see eq. (2.3)) but it is flavour diagonal. The function $g^{(1)}$ indeed depends on the partonic channel that produces the $t \bar{t}$ pair at the LO level. Since $t \bar{t}$ production receives LO contributions from $q \bar{q}$ annihilation and $g g$ fusion, both channels have to be considered. The function $g^{(1)}$ for $t \bar{t}$ production exactly coincides with the corresponding function for DY and Higgs boson production in the $q \bar{q}$ and $g g$ channel, respectively.

Analogously to the production of colourless systems, the functions $g_{N}^{(k)}$ (with $k \geq 2$ ) in eq. (2.5) are matrices in the flavour indices of the partons. Therefore, the practical implementation of the resummation formula (2.5) in exponentiated form requires a proper diagonalization of these matrices with respect to the flavour indices. Such diagonalization and exponentiation procedure in flavour space (see appendix A in ref. [40]) is completely analogous to the customary procedure that is usually applied to the scale evolution of the PDFs.

The main differences between the production of colourless systems and $t \bar{t}$ production start at the NLL level. In the case of $t \bar{t}$ production the functions $g_{N}^{(2)}, g_{N}^{(3)}$ and so forth have an additional component [36-38] due to soft-parton radiation at wide angles with respect to the direction of the colliding partons (hadrons). This component is produced by the non-vanishing colour charge of $t$ and $\bar{t}$ and, as recalled in section 1 , it embodies the effect of soft radiation from the heavy-quark final state and from initial-state and finalstate interferences. Soft wide-angle radiation leads to two main (and related) effects in the structure of the resummation formulae in eqs. (2.4), (2.5) and (2.7): dependence on the scattering angle and presence of colour correlations. The functions $g_{N}^{(k)}$ with $k \geq 2$ in eq. (2.5) acquire a dependence on the scattering angle $\theta$ (such dependence is instead absent in the production of colourless systems) and both these functions and the factor $\mathcal{H}$ in eq. (2.4) are matrices in the colour space of the underlying LO production processes $q \bar{q} \rightarrow t \bar{t}$ and $g g \rightarrow t \bar{t}$ (we recall that the dependence on the colour indices is neglected in the notation of eqs. (2.4) and (2.5)).

The explicit expressions of the NLL+NLO contributions $g_{N}^{(2)}$ and $\mathcal{H}^{(1)}$ in eqs. (2.5) and (2.7) can be worked out from the results in ref. [38]. The term $\mathcal{H}^{(1)}$ (including its depen- 
dence on colour indices) is related to the hard-virtual amplitude $\widetilde{\mathcal{M}}^{(1)}$ in eq. (29) of ref. [38]. The soft wide-angle component of $g_{N}^{(2)}$ and, more generally, of $\mathcal{G}_{N}$ is related to the resummation factor $\mathbf{V}\left(b, M ; y_{34}\right)$ in eqs. (15) and (16) of ref. [38]. The rapidity variable $y_{34}$ of ref. [38] is directly related to the scattering angle $\theta\left(y_{34}=\ln [(1+\beta \cos \theta) /(1-\beta \cos \theta)], \beta=\right.$ $\left.\sqrt{1-4 m_{t}^{2} / M^{2}}\right)$. The resummation factor $\mathbf{V}$ is the exponential of a soft anomalous dimension matrix, $\boldsymbol{\Gamma}_{t}$, in colour space. Therefore, the exponentiated form in eq. (2.5) requires a proper diagonalization procedure [47] of the soft anomalous dimension matrix with respect to its colour indices (such procedure is formally similar to the diagonalization with respect to flavour parton indices that we have previously mentioned). We have explicitly worked out the colour space diagonalization of the one-loop soft anomalous dimension $\boldsymbol{\Gamma}_{t}^{(1)}\left(\mathrm{see}^{2}\right.$ eq. (33) in ref. [38]), whose eigenvalues contribute to the NLL function $g_{N}^{(2)}$ in eq. (2.5). The factor $\mathcal{H}_{N}^{(0)}$ in eq. (2.7) is a colour space matrix and, specifically, the colour space matrix elements of $d \hat{\sigma}_{c \bar{c}}^{(0)} \mathcal{H}_{N}^{(0)}$ are obtained by projecting the LO cross section onto the eigenvectors of the soft anomalous dimension $\boldsymbol{\Gamma}_{t}^{(1)}$ (in the case of production of colourless systems we simply have $\left.\mathcal{H}_{N}^{(0)}=1\right)$.

We recall [40] that the resummed factor $\exp \left\{\mathcal{G}\left(\alpha_{\mathrm{S}}, \tilde{L}\right)\right\}$ of eq. (2.4) is singular at very large values of $b$. The singularity occurs in the region where $b \gtrsim 1 / \Lambda_{\mathrm{QCD}}, \Lambda_{\mathrm{QCD}}$ being the momentum scale of the Landau pole of the perturbative running coupling $\alpha_{\mathrm{S}}\left(\mu^{2}\right)$. This singularity is the 'perturbative' signal of the onset of non-perturbative (NP) phenomena at very large values of $b$ (which practically affect the region of very small transverse momenta). A simple and customary procedure to include NP effects is as follows. The singular behaviour of the perturbative form factor $\exp \left\{\mathcal{G}\left(\alpha_{\mathrm{S}}, \tilde{L}\right)\right\}$ is removed by using a regularization prescription and the resummed expression in eq. (2.4) is then multiplied by a NP form factor and it is inserted as integrand of the $b$ space integral in eq. (2.3). In the present work we use the so called ' $b_{*}$ prescription' of ref. [48], which is obtained by performing the replacement

$$
b^{2} \rightarrow b_{*}^{2}=b^{2} b_{\lim }^{2} /\left(b^{2}+b_{\lim }^{2}\right)
$$

in the $b$ dependence of $\mathcal{G}\left(\alpha_{\mathrm{S}}, \tilde{L}\right)$. The value of the parameter $b_{\text {lim }}$ has to be large $\left(b_{\lim } M \sim b_{\lim } Q \gg 1\right)$ but smaller than the value of $b$ at which the singularity of $\exp \left\{\mathcal{G}\left(\alpha_{\mathrm{S}}, \tilde{L}\right)\right\}$ takes place (note that the replacement in eq. (2.8) has a negligible effect at small and intermediate values of $b$ since $b_{*}^{2}=b^{2}\left(1+\mathcal{O}\left(b^{2} Q^{2} / b_{\lim }^{2} Q^{2}\right)\right) \simeq b^{2}$ if $\left.b Q \lesssim 1\right)$.

\section{Results}

In this section we present the numerical results of our calculation and we compare them to ATLAS and CMS Run 1 data. As stated in section 1, LHC measurements of the transversemomentum distribution of the top-quark pair have been carried out in $p p$ collisions at $\sqrt{s}=7,8$ and $13 \mathrm{TeV}[15]-[24]$. The measurements at $\sqrt{s}=7 \mathrm{TeV}$ are based on data sets with relatively-small integrated luminosity. A similar comment applies to the available

\footnotetext{
${ }^{2}$ A relative sign is mistyped in the right-hand side of the kinematical relation (34) in ref. [38]. The correct result is obtained by performing the replacement $\ln \left(m_{T}^{2} / m^{2}\right) \rightarrow-\ln \left(m_{T}^{2} / m^{2}\right)$ in the right-hand side of eq. (34) therein.
} 
measurements at $\sqrt{s}=13 \mathrm{TeV}$, with the exception of CMS results [24] that have been presented only very recently. In the following we limit ourselves to considering $p p$ collisions at $\sqrt{s}=8 \mathrm{TeV}$. The experimental data that we consider in our comparison are those from ATLAS [17], which correspond to an integrated luminosity of $20.2 \mathrm{fb}^{-1}$ and refer to the dilepton decay channels of the $t \bar{t}$ pair, and those from CMS [18], which correspond to an integrated luminosity of $19.7 \mathrm{fb}^{-1}$ and refer to both the lepton+jets and dilepton channels.

The structure of the resummed cross section is illustrated in section 2. Our resummed calculation is implemented in a numerical program that is obtained as an extension to heavy-quark production of analogous codes for Higgs [49] and vector [45] boson production. We implement the resummed calculation at fixed values of $M$ and $\cos \theta$ (see eq. (2.3)). We limit ourselves to presenting numerical results for the differential cross section $d \sigma / d q_{T}$, which is obtained by numerical integration over $M$ and $\cos \theta$. The regular component of the cross section (see eq. (2.2)) is computed by considering the complete fixed-order result [50] and subtracting the perturbative expansion of the resummed component of the cross section at the corresponding fixed order.

We are going to present resummed results at NLL+NLO accuracy and, for the sake of comparison, we also present fixed-order results for the $q_{T}$ distribution of the $t \bar{t}$ pair up to $\mathcal{O}\left(\alpha_{\mathrm{S}}^{3}\right)$ and $\mathcal{O}\left(\alpha_{\mathrm{S}}^{4}\right)$. The fixed-order results for $d \sigma / d q_{T}$ up to $\mathcal{O}\left(\alpha_{\mathrm{S}}^{3}\right)$ and $\mathcal{O}\left(\alpha_{\mathrm{S}}^{4}\right)$ contribute to the $t \bar{t}$ total cross section at NLO and NNLO, respectively. However, in the region where $q_{T} \neq 0$, they formally ${ }^{3}$ correspond to 'effective' LO [6] and NLO calculations [25-28], respectively. Our results at $\mathcal{O}\left(\alpha_{\mathrm{S}}^{3}\right)$ and $\mathcal{O}\left(\alpha_{\mathrm{S}}^{4}\right)$ are obtained by using the calculation and the numerical program of ref. [50]. The $q_{T}$ cross section at $\mathcal{O}\left(\alpha_{\mathrm{S}}^{4}\right)$ is evaluated by using the Munich code [51], which is also at the heart of the Matrix framework [52]. Munich provides a fully automated implementation of the NLO dipole subtraction formalism [53-55] as well as an interface to the one-loop generator OpenLoops [56] to obtain all the required (spin- and colour-correlated) tree-level and one-loop amplitudes. For the evaluation of tensor integrals we rely on the ColLIER library [57], which is based on the Denner-Dittmaier reduction techniques $[58,59]$ of tensor integrals and on the scalar integrals of ref. [60]. In OpenLoops problematic phase space points are addressed with a rescue system that uses the quadruple-precision implementation of the OPP method in CuTTools [61] with scalar integrals from ONELOOP [62].

To evaluate the normalized $q_{T}$ distribution (see eq. (3.1) below) at NNLO, the NNLO total cross section is required. We compute it by using the numerical program Top $++[63]$, which implements the NNLO calculation of refs. [7-10].

In the following we first focus our discussion on the $q_{T}$ distribution of the $t \bar{t}$ pair at fixed order (section 3.1) and then we move to present our resummed results (section 3.2).

\subsection{Fixed-order results}

We start the presentation of our numerical results by considering QCD calculations at fixed order. To compute the NLO and NNLO hadronic $q_{T}$ cross section (see eq. (2.1)) we use the

\footnotetext{
${ }^{3}$ In spite of the 'effective' meaning of the results in different regions of $q_{T}$, we always use the default labels NLO and NNLO according to the perturbative order in which the results contribute to the total cross section.
} 


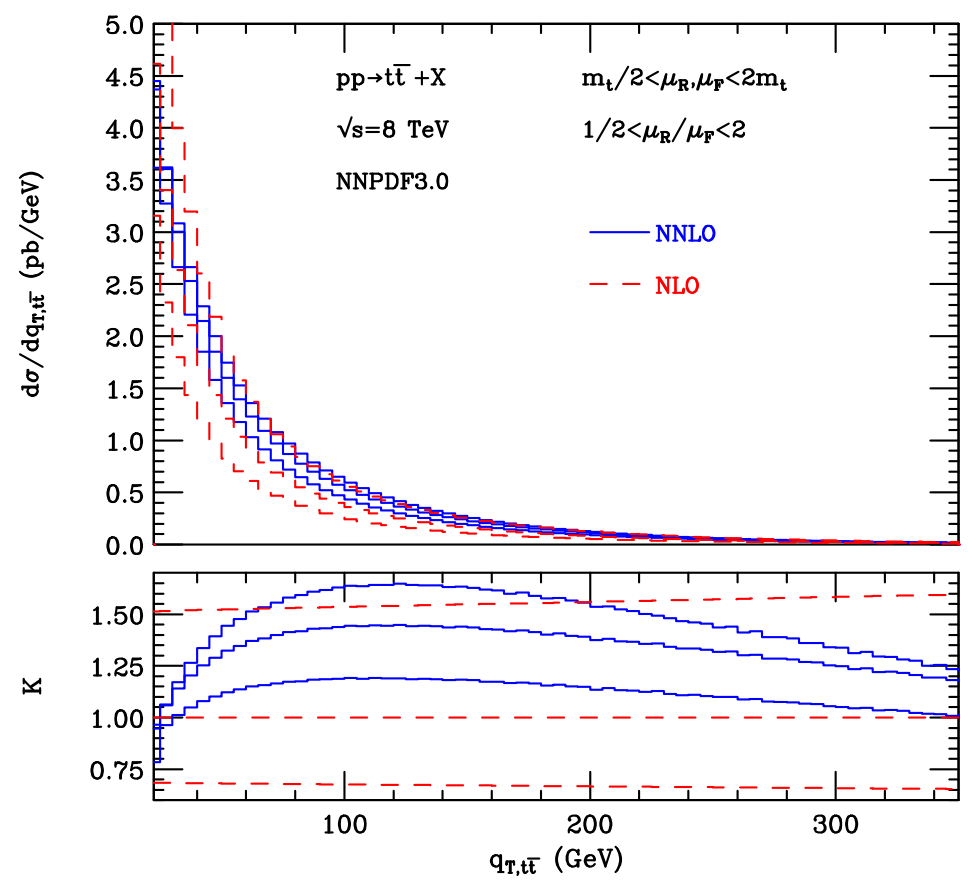

Figure 1. The $q_{T}$ cross section $d \sigma / d q_{T}$ of the $t \bar{t}$ pair produced in $p p$ collisions at $\sqrt{s}=8 \mathrm{TeV}$ : NLO (red dashed) and NNLO (blue solid) theoretical predictions at central scales and including scale dependence. The lower panel shows the ratio $K$ of the NNLO and NLO results (blue solid), and the relative scale dependence at NLO (red dashed).

NNPDF3.0 [64] sets of PDFs at NLO and NNLO with $\alpha_{\mathrm{S}}\left(m_{Z}\right)=0.118$. Correspondingly, the scale $(\mu)$ dependence of the strong coupling constant $\alpha_{\mathrm{S}}\left(\mu^{2}\right)$ is evaluated at two and three loop accuracy in the NLO and NNLO calculations, respectively. The value of the pole mass of the top quark is $m_{t}=173.3 \mathrm{GeV}$. As for the factorization $\left(\mu_{F}\right)$ and renormalization $\left(\mu_{R}\right)$ scales, we choose $\mu_{F}=\mu_{R}=m_{t}$ as central value, and we consider variations of $\mu_{F}$ and $\mu_{R}$ around this central value.

Using these parameters the values of the $t \bar{t}$ total cross section at central scales are $\sigma=224.1 \mathrm{pb}$ at NLO and $\sigma=243.5 \mathrm{pb}$ at NNLO. We note the NNLO corrections increase the NLO total cross section by approximately $9 \%$.

In figure 1 we consider the region where $q_{T}>20 \mathrm{GeV}$ and we present the differential cross section $d \sigma / d q_{T}$ of the $t \bar{t}$ pair at the LHC Run I $(\sqrt{s}=8 \mathrm{TeV})$ at NLO and NNLO accuracy. We present results at central values of the scales and including scale variation effects. The bands are obtained by independently varying the factorization and renormalization scales by a factor ot two around the central values $\mu_{F}=\mu_{R}=m_{t}$ (i.e., we consider the range $0.5 m_{t} \leq\left\{\mu_{F}, \mu_{R}\right\} \leq 2 m_{t}$ ), with the constraint $0.5 \leq \mu_{F} / \mu_{R} \leq 2$. At the NLO the scale dependence is at the level of roughly $\pm 40 \%$. At NNLO the scale dependence is relatively flat in the intermediate region $80 \mathrm{GeV} \leq q_{T} \leq 200 \mathrm{GeV}$ and it is about $\pm 18 \%$. The NNLO scale dependence slightly decreases at larger values of $q_{T}$ and it also decreases at smaller values of $q_{T}$. 


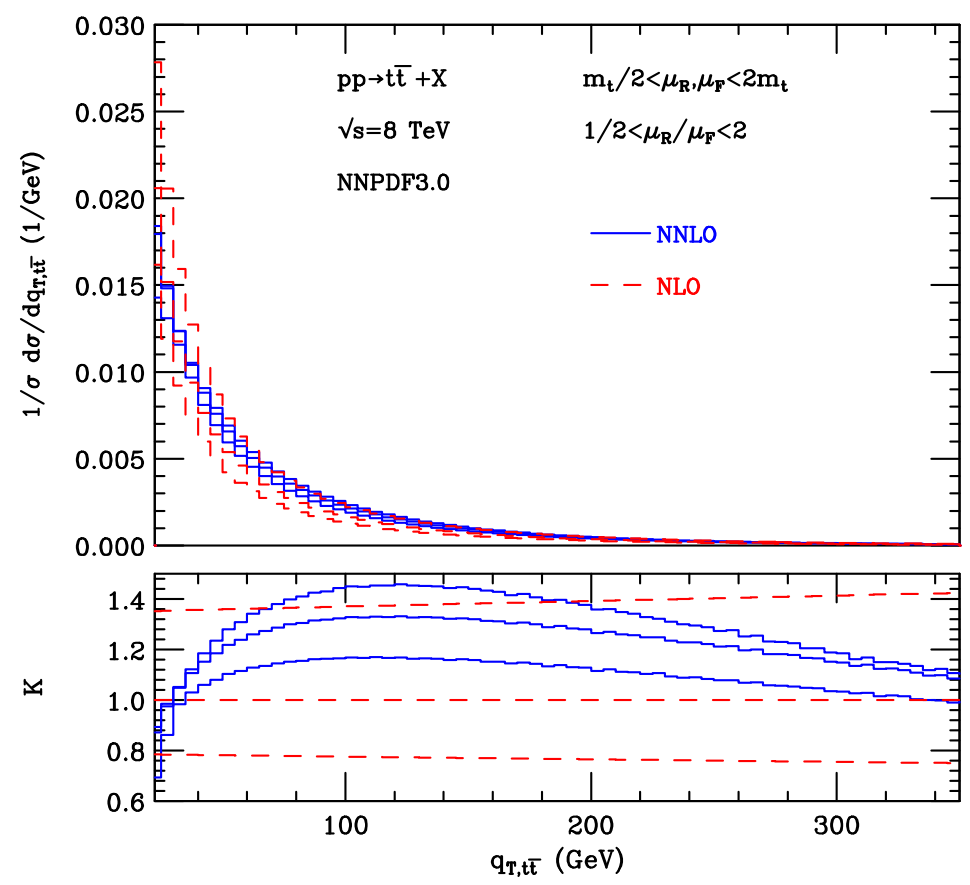

Figure 2. Same as in figure 1 for the normalized $q_{T}$ distribution $1 / \sigma\left(d \sigma / d q_{T}\right)$.

The lower panel of figure 1 shows the $K$ factor, which is obtained by normalizing the NNLO band with respect to the NLO result at $\mu_{F}=\mu_{R}=m_{t}$. Using the same normalization we also show the scale dependence of the NLO result. At central values of the scales the impact of the NNLO corrections ranges from about $-5 \%$ at $q_{T} \sim 20 \mathrm{GeV}$ to about $+18 \%$ at $q_{T} \sim 350 \mathrm{GeV}$. The $K$ factor is relatively flat and larger in the intermediate $q_{T}$ region $\left(80 \mathrm{GeV} \leq q_{T} \leq 160 \mathrm{GeV}\right)$ where its value is about 1.4 .

As shown in figure 1 , at intermediate and large values of $q_{T}$ the scale variation bands of the NLO and NNLO results overlap and the NNLO result has a reduced scale dependence. In this $q_{T}$ region the NNLO scale dependence can consistently be used as an approximate estimate of the theoretical uncertainty of the NNLO prediction. At smaller values of $q_{T}$ (say, $q_{T} \lesssim 60 \mathrm{GeV}$ ), the NNLO scale dependence strongly decreases by decreasing $q_{T}$ and the size of the $K$ factor becomes close to unity at $q_{T} \sim 30 \mathrm{GeV}$. We anticipate (see the discussion of the results in figure 3-right) that the behaviour of the NNLO radiative corrections at $q_{T} \sim 30 \mathrm{GeV}$ should not be regarded as a signal of perturbative convergence: in constrast, it is just a consequence and an artifact of the order-by-order perturbative instability of the shape of the $q_{T}$ cross section in the small- $q_{T}$ region.

The results in figure 2 are analogous to those in figure 1, but they refer to the normalized $q_{T}$ spectrum of the $t \bar{t}$ pair. We consider the normalized $q_{T}$ distribution, $\frac{1}{\sigma} \frac{d \sigma}{d q_{T}}$, of $t \bar{t}$ pairs at the LHC Run I $(\sqrt{s}=8 \mathrm{TeV})$ at NLO and NNLO accuracy. More precisely, we define

$$
\left(\frac{1}{\sigma} \frac{d \sigma}{d q_{T}}\right)_{(\mathrm{N}) \mathrm{NLO}}\left(\mu_{F}, \mu_{R}\right) \equiv \frac{1}{\sigma_{(\mathrm{N}) \mathrm{NLO}}\left(\mu_{F}, \mu_{R}\right)}\left(\frac{d \sigma}{d q_{T}}\right)_{(\mathrm{N}) \mathrm{NLO}}\left(\mu_{F}, \mu_{R}\right)
$$


and the two factors, $1 / \sigma$ and $d \sigma / d q_{T}$, on the right-hand side of eq. (3.1) are evaluated by using the same PDFs and the same values of the renormalization and factorization scales. The scale variation bands and the $K$ factor are obtained as in the case of the cross section results of figure 1 .

From the results in figure 2, we see that the NLO scale dependence of the normalized distribution is at the level of roughly $\pm 30 \%$. At NNLO the scale dependence is about $\pm 12 \%$ in the region where $80 \mathrm{GeV} \leq q_{T} \leq 200 \mathrm{GeV}$. At central values of the scales the impact of the NNLO corrections (lower panel in figure 2) ranges from about $-10 \%$ at $q_{T} \sim 20 \mathrm{GeV}$ to about $+10 \%$ at $q_{T} \sim 350 \mathrm{GeV}$. The $K$ factor is relatively flat and larger in the intermediate $q_{T}$ region $\left(80 \mathrm{GeV} \leq q_{T} \leq 160 \mathrm{GeV}\right)$ where its value is about 1.3 .

Comparing the results in figure 1 with those in figure 2, we can see that they have very similar features. The main differences are that the results for the normalized $q_{T}$ distribution have a decreased scale dependence (at both NLO and NNLO) and a smaller NNLO $K$ factor in the intermediate $q_{T}$ region. This implies that QCD radiative corrections to $d \sigma / d q_{T}$ include contributions with a small dependence on $q_{T}$ that do not affect the shape of the $q_{T}$ spectrum. Their effect and the related scale dependence partly cancel in the ratio between $d \sigma / d q_{T}$ and the total cross section $\sigma$.

In figure 3 we compare the fixed-order predictions for the normalized $q_{T}$ distribution with the experimental data from ATLAS [17] and CMS [18]. The plots in figure 3 show both the NLO and NNLO predictions with their scale uncertainties normalised to the NNLO result at central values of the scales, and the relative deviation of the data from such central NNLO prediction. Thus the results presented in figure 3 refer to the fractional differences (X-'theory')/'theory', where $\mathrm{X}=\{\mathrm{NLO}, \mathrm{NNLO}$, data $\}$ and the reference theoretical result ('theory') is the NNLO prediction at central scales. We point out that the theoretical predictions are obtained by using $q_{T}$ bins with a constant size of $5 \mathrm{GeV}$, while the comparison to the data is done by using exactly the same bin sizes that are used in the experimental measurements. The two panels in figure 3 present the same content by using either a linear (left panel) or a logarithmic (right panel) $q_{T}$ scale on the horizontal axis. The region where $q_{T}<20 \mathrm{GeV}\left(q_{T}>150 \mathrm{GeV}\right)$ is excluded in the left (right) panel.

We start our discussion of the results in figure 3 by considering the region of intermediate and high values of $q_{T}$ (left panel). Figure 3-left and the lower panel of figure 2 present the same fixed-order results, but the role of the NNLO and NLO central values as reference theoretical result is exchanged between the two figures, and the overall shapes of the NLO and NNLO bands are exchanged accordingly. At intermediate and large values of $q_{T}$ we see that the data are consistent with the NNLO prediction within uncertainties. Only two data points overshoot the NNLO prediction, but the difference is smaller than one standard deviation. We also note that the experimental uncertainties and the NNLO uncertainties, estimated through scale variations, are of similar size. The data and the NLO predictions are not inconsistent within uncertainties. The NNLO corrections improve the agreement with the data by both increasing the central value of the theoretical prediction and decreasing its scale uncertainty.

We now move to consider the small- $q_{T}$ region (figure 3-right). We first discuss the behaviour of the fixed-order results. Starting from $q_{T} \sim 60 \mathrm{GeV}$ and decreasing the value of $q_{T}$, the NNLO scale dependent band shrinks, by reaching a minimum size around $q_{T} \sim$ 


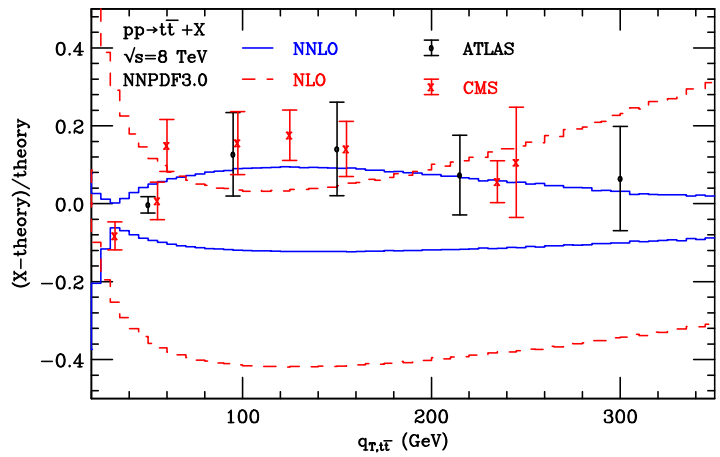

(a)

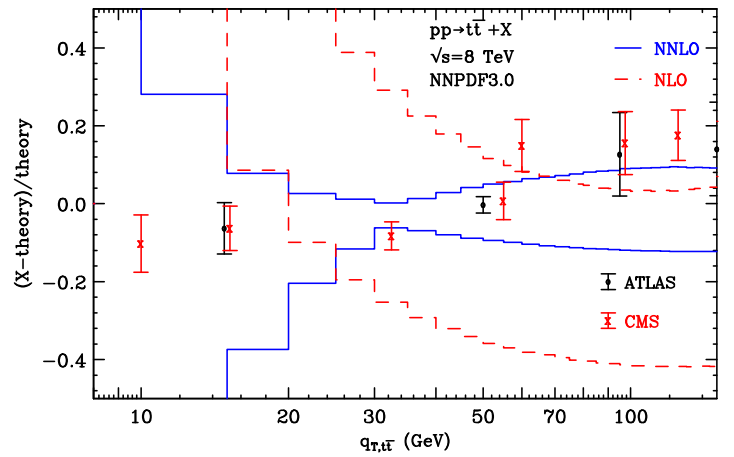

(b)

Figure 3. Fractional difference of NLO predictions (red dashed), NNLO predictions (blue solid) and LHC data $[17,18]$ with respect to the NNLO result at $\mu_{F}=\mu_{R}=m_{t}$. The results refer to the normalized $q_{T}$ distribution $1 / \sigma\left(d \sigma / d q_{T}\right)$ of $t \bar{t}$ pairs at $\sqrt{s}=8 \mathrm{TeV}$. The two panels highlight (a) the intermediate and large $q_{T}$ region and (b) the small- $q_{T}$ region.

$30 \mathrm{GeV}$, where the ratio NLO/NNLO at central scales is close to unity. Then, by further decreasing $q_{T}$, the size of the NNLO band quickly increases and the NNLO scale dependence is of the order of $\pm 50 \%$ or larger at $q_{T} \sim 10 \mathrm{GeV}$. Moreover, in the region below $q_{T} \sim$ $20 \mathrm{GeV}$, the NLO band quickly departs from the NNLO band and the ratio NLO/NNLO at central scales suddenly becomes much different from unity: in this $q_{T}$ region the fixed-order expansion cannot reliably predict the detailed shape of the $q_{T}$ spectrum. At $q_{T} \sim 30 \mathrm{GeV}$, the fixed-order expansion is apparently quite stable but this stability has to be regarded as partly accidental since it takes place very close to the low- $q_{T}$ region where the convergence of the fixed-order expansion is spoiled.

The behaviour of the NLO and NNLO results at small $q_{T}$ is produced by the large logarithmic terms that we have discussed in sections 1 and 2 . The qualitative features of the NLO and NNLO results in figure 3-right are indeed completely similar to those of analogous fixed-order results for other processes that are affected by large logarithmic contributions in the small $-q_{T}$ region (see, e.g., figure 3 in ref. [65] and accompanying comments for a related discussion in the context of vector boson production).

In the small- $q_{T}$ region the perturbative resummation of the large logarithmic terms is necessary to reliably predict the detailed shape of the $q_{T}$ spectrum of the $t \bar{t}$ pair. Nonetheless, we note that the data points in figure 3-right are still perfectly consistent with the NNLO result within uncertainties. This is a consequence of the fact that the experimental measurements use relatively-large bin sizes in $q_{T}$ : the first bin covers the region up to $q_{T}=20 \mathrm{GeV}$ for the CMS lepton+jets data set and up to $q_{T}=30 \mathrm{GeV}$ for the other two measurements (in figure 3 each data point is placed at the midpoint of the corresponding $q_{T}$ bin). Using such relatively-large bin sizes, the singular behavior of the fixed-order calculation at $q_{T} \rightarrow 0$ is smeared out, and it turns out that at NNLO a sensible central result ${ }^{4}$ is obtained, though it is affected by very large scale uncertainties.

\footnotetext{
${ }^{4}$ We remark that in the lowest- $q_{T}$ bin (which includes $q_{T}=0$ ) the NNLO result for $1 / \sigma\left(d \sigma / d q_{T}\right)$ includes the complete contributions at the first three perturbative orders (i.e., $\left.\mathcal{O}\left(\alpha_{\mathrm{S}}^{2}+\alpha_{\mathrm{S}}^{3}+\alpha_{\mathrm{S}}^{4}\right)\right)$ in the computation of both $d \sigma / d q_{T}$ and $\sigma$.
} 
In our comments throughout this subsection we have made distinctions among small, intermediate and large values of $q_{T}$. We notice that the $q_{T}$ spectrum of $t \bar{t}$ pairs at the LHC is quite broad and it has an average transverse momentum $\left\langle q_{T}\right\rangle$ of approximately $50 \mathrm{GeV}$. At the NLO with central value of the scales we have $\left\langle q_{T}\right\rangle=50.1 \mathrm{GeV}$. We note that such value of $\left\langle q_{T}\right\rangle$ is roughly three times larger than the NLO value [66] for $t \bar{t}$ production at the Tevatron $(p \bar{p}$ collisions at $\sqrt{s}=1.8 \mathrm{TeV})$. The value of $\left\langle q_{T}\right\rangle$ is approximately given by the proportionality relation $\left\langle q_{T}\right\rangle \sim C \alpha_{\mathrm{S}} m_{t}$, where the proportionality factor $C$ depends on the underlying QCD dynamics and it has a weak dependence on $\sqrt{s}(C$ slowly increases by increasing $\sqrt{s}$ because of the larger available phase space). The contribution to $C$ from initial-state radiation tends to be proportional to the colour coefficient $C_{A}=N_{c}=$ $3\left(C_{F}=\left(N_{c}^{2}-1\right) /\left(2 N_{c}\right)=4 / 3\right)$ for production subprocesses that are due to $g g$ fusion ( $q \bar{q}$ annihilation). This colour coefficient dependence qualitatively explains why the $q_{T}$ spectrum at the LHC is much broader than the spectrum at the Tevatron. Indeed, the $t \bar{t}$ pair is mostly produced by $g g$ fusion at the LHC, whereas $q \bar{q}$ annihilation dominates at the Tevatron (this is a consequence of the relative differences between $g g$ and $q \bar{q}$ PDF luminosities in $p p$ collisions at the LHC and $p \bar{p}$ collisions at the Tevatron).

\subsection{Resummed results}

In the following we present our resummed results at NLL+NLO accuracy and we compare them with the LHC data. To compute the resummed cross sections, we use the NNPDF3.0 NLO PDFs [64], with the scale dependence of $\alpha_{\mathrm{S}}\left(\mu^{2}\right)$ evaluated at two-loop order. The pole mass of the top quark is $m_{t}=173.3 \mathrm{GeV}$ as in our fixed-order calculations. As discussed in section 2, the resummed predictions depend on renormalization, factorization and resummation scales. The effect of factorization and renormalization scale variations is computed as in the fixed-order calculations of section 3.1 by using $\mu_{F}=\mu_{R}=m_{t}$ as central value of these scales. We choose $Q=m_{t}$ as central value of the resummation scale $Q$, and we consider resummation scale variations in the range $m_{t} / 2<Q<2 m_{t}$. The parameter $b_{\lim }$ in eq. (2.8) is set to the value $b_{\lim }=3 \mathrm{GeV}^{-1}$.

We note that the auxiliary scales $\mu_{F}, \mu_{R}$ and $Q$ have to be chosen of the order of the typical hard scale of the cross section to avoid a parametrically-large scale dependence from missing higher-order contributions (in the context of both fixed-order and resummed perturbation theory). In the case of the $q_{T}$ spectrum at fixed invariant mass $M$ of the $t \bar{t}$ pair (section 2), the typical hard scale of the cross section is $M$. In the case of the $q_{T}$ spectrum integrated over $M$ (which is considered in all the results of section 3), the typical hard scale turns out to be of the order of $m_{t}$ since the bulk of the invariant mass distribution is concentrated within a narrow region where $M \sim 2 m_{t}$ (see e.g. figure 8 in ref. [13]).

In figure 4 the NLL+NLO cross section $d \sigma / d q_{T}$ (solid line) at the central scales ( $\mu_{F}=$ $\mu_{R}=Q=m_{t}$ ) is compared with the corresponding NLO result (dashed line) and with the regular component $d \sigma^{(\mathrm{reg})} / d q_{T}$ (see eq. (2.2)) of the cross section (dotted line). At small values of $q_{T}$ the NLO result becomes arbitrarily large by decreasing $q_{T}$ towards lower (non-vanishing) values. In the first $q_{T}$-bin of figure $4\left(q_{T} \leq 5 \mathrm{GeV}\right)$, which includes $q_{T}=0$, the NLO result is negative (the negative value is outside the vertical scale of the plot in 


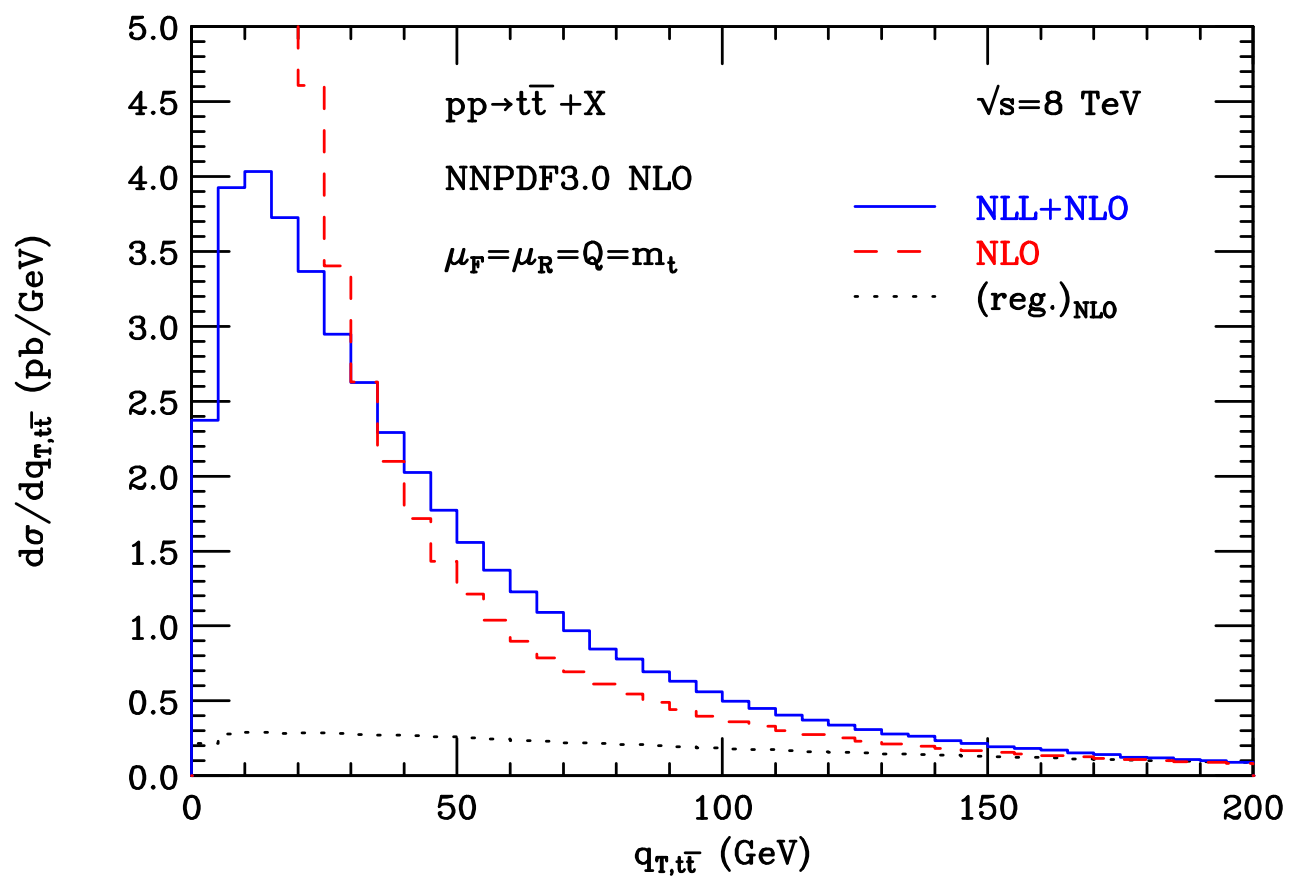

Figure 4. The transverse-momentum cross section $d \sigma / d q_{T}$ of the $t \bar{t}$ pair at the $\mathrm{LHC}(\sqrt{s}=8 \mathrm{TeV})$ computed through resummation at NLL+NLO accuracy (blue solid). The resummed result at central scales $\left(\mu_{R}=\mu_{F}=Q=m_{t}\right)$ is compared to the corresponding NLO result (red dashed). The contribution of the regular component (black dotted) to the NLL+NLO result is also shown.

figure 4). Such behaviour of the NLO result is definitely unphysical. The resummation of the small- $q_{T}$ logarithms leads to a physically well-behaved distribution at small transverse momenta, with a kinematical ${ }^{5}$ peak in the region where $q_{T} \sim 10 \mathrm{GeV}-15 \mathrm{GeV}$. At large values of $q_{T}$, the NLL+NLO result tends to the corresponding NLO result. In the small- $q_{T}$ region the NLL+NLO result is dominated by resummation, although the contribution of the regular component is not negligible (it is approximately $9 \%$ ). In the region of intermediate values of $q_{T}$ (say, around $100 \mathrm{GeV}$ ), the contribution of the regular component increases to about $35 \%$ of the NLL+NLO result. At larger values of $q_{T}$ the contribution of the regular component sizeably increases, indicating that the logarithmic terms are no longer dominant and that the resummed calculation cannot improve upon the predictivity of the fixed-order expansion.

In figure 5 we show the scale dependence of our resummed results.

In figure 5-left we consider the effect of variations of the renormalization and factorization scales by keeping the resummation scale fixed at the central value $Q=m_{t}$. The bands are obtained by independently varying $\mu_{R}$ and $\mu_{F}$ as usually done throughout this paper. The scale variation in the peak region is at the level of about $\pm 20 \%$. At intermediate values of $q_{T}$ the scale variation band shrinks a bit, while in the region of large values of $q_{T}$ the scale dependence increases dramatically, reaching even the level of $\pm 100 \%$.

\footnotetext{
${ }^{5}$ After resummation $d \sigma / d q_{T}^{2} \rightarrow$ const. as $q_{T} \rightarrow 0$. Therefore, $d \sigma / d q_{T}=2 q_{T} d \sigma / d q_{T}^{2}$ vanishes as $q_{T} \rightarrow 0$ and it has a peak due to the kinematical (Jacobian) factor of $2 q_{T}$.
} 


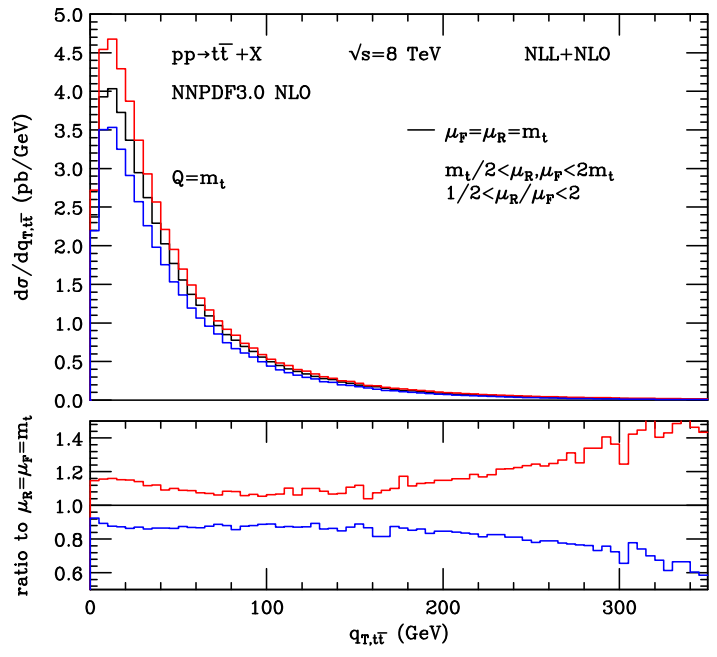

(a)

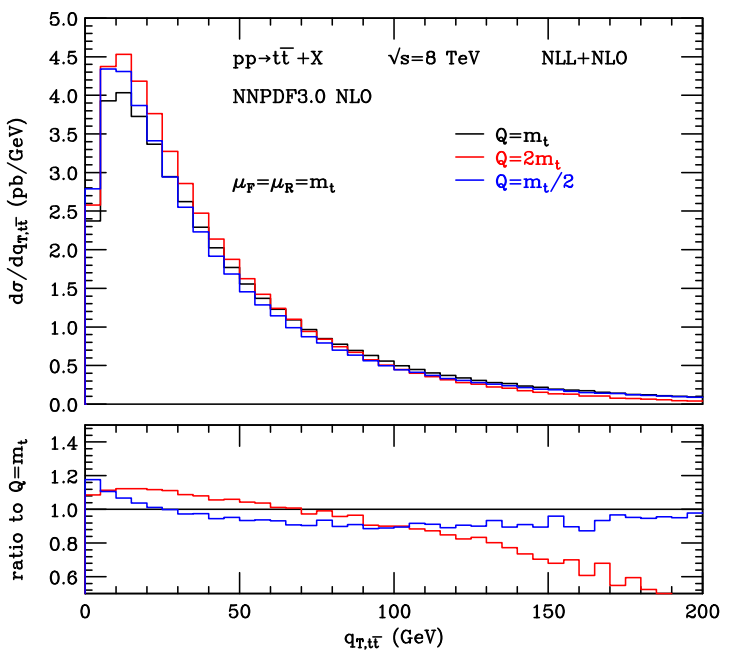

(b)

Figure 5. The transverse-momentum cross section $d \sigma / d q_{T}$ of the $t \bar{t}$ pair at the $\mathrm{LHC}(\sqrt{s}=8 \mathrm{TeV})$ computed at NLL+NLO accuracy. The bands (blue and red lines) are obtained by varying $\mu_{F}$ and $\mu_{R}$ (left) and $Q$ (right) as described in the text. The lower panels present the scale variation bands relative to the result at central scales $\left(\mu_{R}=\mu_{F}=Q=m_{t}\right)$.

In figure 5-right we consider the effect of resummation scale variations. The results are obtained by fixing $\mu_{R}=\mu_{F}=m_{t}$ and considering three values $Q=\left\{m_{t} / 2, m_{t}, 2 m_{t}\right\}$ of the resummation scale $Q$. Performing variations of the resummation scale, we can get further insight on the size of yet uncalculated higher-order logarithmic contributions at small and intermediate values of $q_{T}$. In the peak region we find that the resummation scale dependence is about $15 \%$ and positive. Resummation scale effects of similar size are found at intermediate values of $q_{T}$, while at very large transverse momenta the resummation scale dependence is negative and very large.

As recalled in section 2, our resummation procedure for the $q_{T}$ cross section formally reproduces the fixed-order result for the total cross section. We find that the integral over $q_{T}$ of our resummed NLL+NLO result of the $q_{T}$ spectrum is in agreement (for any values of $\mu_{R}, \mu_{F}$ and $Q$ ) with the value of the NLO total cross section to better than $1 \%$, thus checking the numerical accuracy of the code. For example, at central values of the scales, the value of our total cross section at NLL+NLO accuracy is $223.8 \mathrm{pb}$, which is in excellent agreement with the value $224.1 \mathrm{pb}$ of the NLO result. We also notice that the average transverse momentum $\left\langle q_{T}\right\rangle$ is little affected by the resummation procedure. At central scales, using the resummed result at NLL+NLO accuracy we obtain $\left\langle q_{T}\right\rangle=51.3 \mathrm{GeV}$, which is very similar to the NLO value $50.1 \mathrm{GeV}$.

At large values of $q_{T}^{6}$ the resummation of the logarithmic terms $\ln \left(q_{T} / M\right)$ is theoretically unjustified, since these terms are not the dominant radiative corrections in the large- $q_{T}$ region. Indeed, as previously observed in our comments on the results in figure 4 ,

\footnotetext{
${ }^{6}$ We notice that the large- $q_{T}$ region gives a very small contribution to the total cross section.
} 
the fixed-order contribution of the regular component $d \sigma^{(\mathrm{reg})} / d q_{T}$ to the $q_{T}$ cross section is sizeable at large values of $q_{T}$. The scale dependence of the NLL+NLO result is larger than the scale dependence of the fixed-order result (though the two results are consistent within scale uncertainties) at large $q_{T}$, and this fact further indicates that resummation is less predictive than the fixed-order expansion. In the large- $q_{T}$ region the resummed prediction can simply be replaced by fixed-order predictions. Alternatively, a 'smooth switching procedure' between the resummed and fixed-order results (see, e.g., refs. [49] and [45]) can consistently be implemented at large values of $q_{T}$.

The NLL+NLO result has (at the formal level) a uniform theoretical accuracy throughout the region from small to intermediate values of $q_{T}$. As discussed in ref. [40], this is the consequence of the consistent combination (matching procedure) of the fixed-order term $d \sigma^{(\mathrm{reg})}$ with the resummed contribution to $d \sigma^{(\mathrm{sing})}$ (see eq. (2.2)) and of the fact that the resummed calculation returns the fixed-order value of the total cross section after integration over $q_{T}$.

A direct quantitative comparison between fixed-order and resummed results at intermediate values of $q_{T}$ is presented in figure 6 . We consider $d \sigma / d q_{T}$ and in figure 6 we present the fractional difference of the scale dependent NLO (dashed), NNLO (solid) and NLL+NLO (dot dashed) results with respect to the NNLO result at central scales $\left(\mu_{F}=\mu_{R}=m_{t}\right)$. The scale dependence of the NLO and NNLO results is obtained by the seven-point scale variation of $\mu_{R}$ and $\mu_{F}$. Therefore, the NNLO and NLO bands in figure 6 exactly corresponds to the bands in figure 1 (though the role of the NNLO and NLO central values as reference theoretical result is exchanged between the two figures). The scale dependence of the NLL+NLO result is obtained by taking the envelope of the seven-point scale variation of $\mu_{R}$ and $\mu_{F}$ at fixed resummation scale $Q=m_{t}$ (see figure 5-left) and the variation of $Q$ by a factor of two at fixed $\mu_{R}=\mu_{F}=m_{t}$ (see figure 5 -right).

In figure 6 we can see that the scale dependence of the NLL+NLO result has a moderate size at intermediate values of $q_{T}$. Moreover, in the region where $50 \mathrm{GeV} \lesssim q_{T} \lesssim 150 \mathrm{GeV}$ the NLL+NLO and NNLO central values are quite close, their difference being always smaller than $10 \%$, and they have comparable scale dependence. We conclude that the NLL+NLO and NNLO results are fully consistent in this intermediate region of transverse momenta. The NLL+NLO calculation provides us with a QCD prediction that can be extended down to lower values of $q_{T}$ with a relatively-small perturbative uncertainty. In particular, in the region where $20 \mathrm{GeV} \lesssim q_{T} \lesssim 50 \mathrm{GeV}$ the NLL+NLO scale dependence is approximately constant and the central value of the NNLO result tends to deviate from the NNLO result. This behaviour is a further indication that the reduction of the NNLO scale dependence at $q_{T} \sim 30 \mathrm{GeV}$ is accidental and it does underestimate the theoretical uncertainty of the NNLO result.

In figure 7 we compare our resummed predictions at NLL+NLO accuracy with the LHC data. The presentation of the results in figure 7 is analogous to that of the corresponding fixed-order comparison in figure 3. The plots in figure 7 show the fractional differences (X'theory')/'theory', where $\mathrm{X}$ is either the NLL+NLO prediction with its scale uncertainty (which is computed as in the results of figure 6) or the LHC data [17, 18], and the reference 


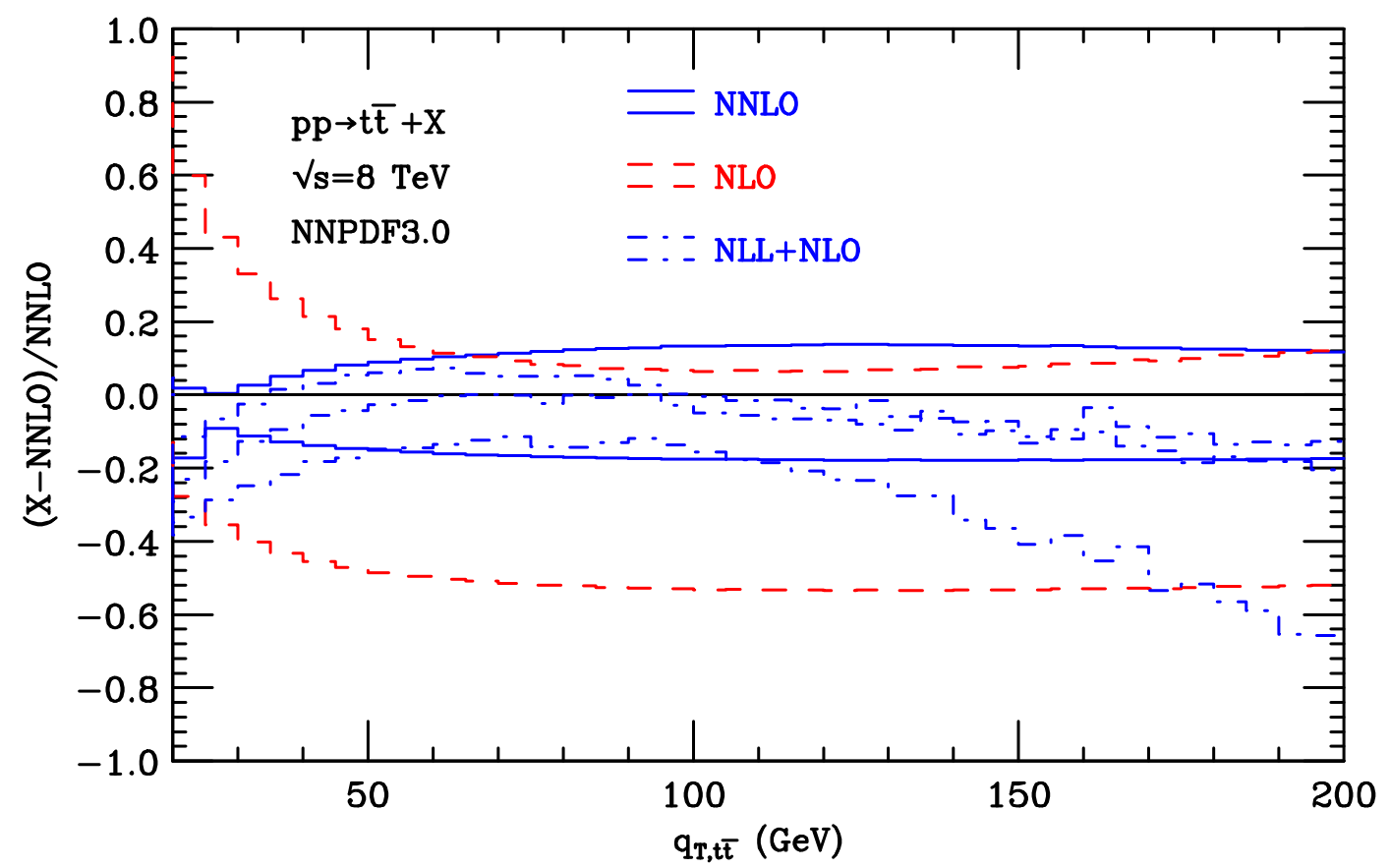

Figure 6. Fractional difference of the scale dependent NLO (red dashed), NNLO (blue solid) and NLL+NLO (blue dot-dashed) results with respect to the NNLO result at $\mu_{F}=\mu_{R}=m_{t}$. The results refer to the differential cross section $d \sigma / d q_{T}$ of $t \bar{t}$ pairs at $\sqrt{s}=8 \mathrm{TeV}$.

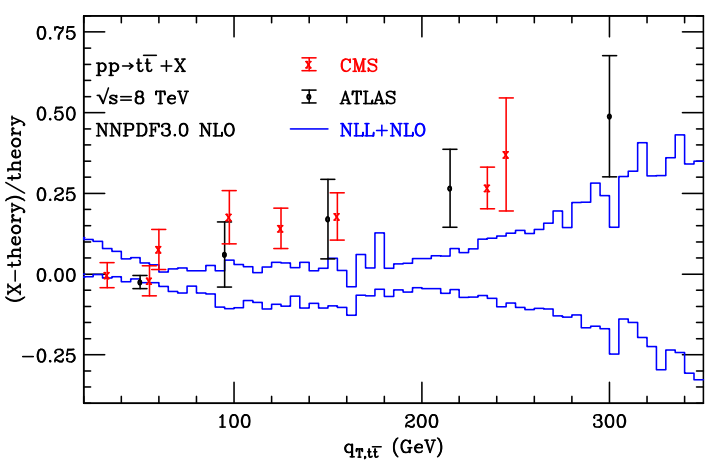

(a)

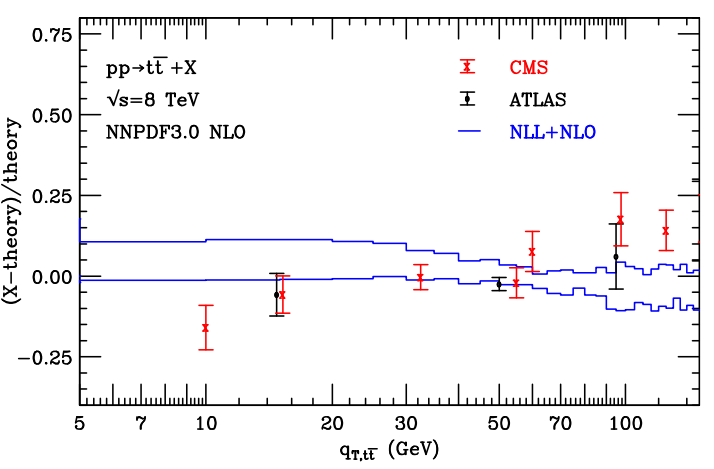

(b)

Figure 7. Fractional difference of scale dependent NLL+NLO prediction and LHC data [17, 18] with respect to the NLL+NLO result at $\mu_{F}=\mu_{R}=Q=m_{t}$. The results refer to the normalized $q_{T}$ distribution $1 / \sigma\left(d \sigma / d q_{T}\right)$ of $t \bar{t}$ pairs at $\sqrt{s}=8 \mathrm{TeV}$. The two panels highlight (a) the intermediate and large $q_{T}$ region and (b) the small- $q_{T}$ region. 


\begin{tabular}{|c|c|c|}
\hline$q_{T, t \bar{t}}[\mathrm{GeV}]$ & $\frac{1}{\sigma} \frac{d \sigma}{d q_{T, t \bar{t}}}\left[\mathrm{TeV}^{-1}\right]$ ATLAS & $\frac{1}{\sigma} \frac{d \sigma}{d q_{T, t \bar{t}}}\left[\mathrm{TeV}^{-1}\right]$ NLL+NLO \\
\hline $0-30$ & $14.3 \pm 1.0$ & $15.2_{-0.3}^{+1.4}$ \\
\hline $30-70$ & $7.60 \pm 0.16$ & $7.79_{-0.17}^{+0.38}$ \\
\hline $70-120$ & $2.94 \pm 0.28$ & $2.77_{-0.21}^{+0.05}$ \\
\hline $120-180$ & $1.14 \pm 0.12$ & $0.97_{-0.09}^{+0.03}$ \\
\hline $180-250$ & $0.42 \pm 0.04$ & $0.33_{-0.02}^{+0.02}$ \\
\hline $250-350$ & $0.143 \pm 0.018$ & $0.096_{-0.015}^{+0.021}$ \\
\hline
\end{tabular}

Table 1. The normalized $q_{T}$ distribution $1 / \sigma\left(d \sigma / d q_{T}\right)$ of $t \bar{t}$ pairs in $p p$ collisions at the LHC $(\sqrt{s}=8 \mathrm{TeV})$ : comparison between ATLAS data (dilepton channels) [17] and NLL+NLO results.

theoretical result ('theory') is the NLL+NLO prediction at central scales $\left(\mu_{F}=\mu_{R}=Q=\right.$ $\left.m_{t}\right)$. As in the case of figure 3 , the NLL+NLO results are obtained by using $q_{T}$ bins with a constant size of $5 \mathrm{GeV}$, while the comparison to the data is done by using exactly the same bin sizes that are used in the experimental measurements. The bin-by-bin comparison between data and NLL+NLO results is explicitly reported also in tables 1, 2 and 3.

The region of small and intermediate values of $q_{T}$ is highlighted in figure 7-right. Throughout the low- $q_{T}$ region the NLL+NLO result has a scale uncertainty that is uniformly of the order of about $\pm 10 \%$. This should be contrasted with the behaviour at fixed order (see figure 3-right), since the (NLO) NNLO scale uncertainty dramatically increases as $q_{T} \rightarrow 0$. In the low- $q_{T}$ region we also see (figure 7 -right and tables $1-3$ ) that the NLL+NLO prediction is consistent with the data within the corresponding uncertainties. Only the first bin of the CMS lepton+jets measurement (table 2) is outside the scale uncertainty band. In the intermediate- $q_{T}$ region (say, in the range $50 \mathrm{GeV} \lesssim q_{T} \lesssim 150 \mathrm{GeV}$ ) data and NLL+NLO results are consistent. The degree of consistency is comparable to that between data and NNLO results (figure 3). This is a consequence of the fact (see comments on figure 6) that NLL+NLO and NNLO results behave similarly at intermediate values of $q_{T}$.

At high values of $q_{T}$ (figure 7-left) the data tend to systematically overshoot the NLL+NLO results. As we have previously noticed, in this $q_{T}$ region the resummed calculation cannot improve the predictivity of the fixed-order expansion, which has to be preferred to obtain QCD predictions. At high $q_{T}$, the agreement with the data definitely improves by replacing the NLL+NLO results with the fixed-order results at NNLO accuracy (figure 3-left).

In section 2 we have recalled and noticed that a distinctive feature of transversemomentum resummation for heavy-quark production is the appearance of dynamical colour-correlation effects in the resummed form factor $\exp \mathcal{G}$ (see eq. (2.4)). These effects are due to soft-parton radiation from the $t \bar{t}$ pair, and they start to contribute at the NLL level through a soft wide-angle component of the function $g_{N}^{(2)}$ in eq. (2.5). It is of interest to quantify the impact of these effects. To this purpose we consider the NLL+NLO calculation of the spectrum by removing the contribution of the soft wide-angle component to the resummed exponent of eq. (2.5) (this is equivalent to set $\boldsymbol{\Gamma}_{t}^{(1)}=0, \boldsymbol{\Gamma}_{t}^{(1)}$ being the 


\begin{tabular}{|c|c|c|}
\hline$q_{T, t \bar{t}}[\mathrm{GeV}]$ & $\frac{1}{\sigma} \frac{d \sigma}{d q_{T, t \bar{t}}}\left[\mathrm{TeV}^{-1}\right] \mathrm{CMS}$ & $\frac{1}{\sigma} \frac{d \sigma}{d q_{T, t \bar{t}}}\left[\mathrm{TeV}^{-1}\right] \mathrm{NLL}+\mathrm{NLO}$ \\
\hline $0-20$ & $13.2 \pm 1.1$ & $15.7_{-0.2}^{+1.9}$ \\
\hline $20-45$ & $11.8 \pm 0.5$ & $11.8_{-0.1}^{+1.0}$ \\
\hline $45-75$ & $6.40 \pm 0.37$ & $5.95_{-0.20}^{+0.17}$ \\
\hline $75-120$ & $2.84 \pm 0.20$ & $2.41_{-0.02}^{+0.23}$ \\
\hline $120-190$ & $1.07 \pm 0.07$ & $0.91_{-0.08}^{+0.03}$ \\
\hline $190-300$ & $0.306 \pm 0.039$ & $0.223_{-0.018}^{+0.022}$ \\
\hline
\end{tabular}

Table 2. The normalized $q_{T}$ distribution $1 / \sigma\left(d \sigma / d q_{T}\right)$ of $t \bar{t}$ pairs in $p p$ collisions at the LHC $(\sqrt{s}=8 \mathrm{TeV})$ : comparison between CMS data (lepton+jets channels) [18] and NLL+NLO results.

\begin{tabular}{|c|c|c|}
\hline$q_{T, t \bar{t}}[\mathrm{GeV}]$ & $\frac{1}{\sigma} \frac{d \sigma}{d q_{T, t \bar{t}}}\left[\mathrm{TeV}^{-1}\right] \mathrm{CMS}$ & $\frac{1}{\sigma} \frac{d \sigma}{d q_{T, t \bar{t}}}\left[\mathrm{TeV}^{-1}\right] \mathrm{NLL}+\mathrm{NLO}$ \\
\hline $0-30$ & $14.3 \pm 0.9$ & $15.2_{-0.2}^{+1.8}$ \\
\hline $30-80$ & $6.9 \pm 0.3$ & $7.0_{-0.2}^{+0.3}$ \\
\hline $80-170$ & $1.91 \pm 0.11$ & $1.67_{-0.15}^{+0.04}$ \\
\hline $170-300$ & $0.347 \pm 0.018$ & $0.274_{-0.002}^{+0.023}$ \\
\hline
\end{tabular}

Table 3. The normalized $q_{T}$ distribution $1 / \sigma\left(d \sigma / d q_{T}\right)$ of $t \bar{t}$ pairs in $p p$ collisions at the LHC $(\sqrt{s}=8 \mathrm{TeV})$ : comparison between CMS data (dilepton channels) [18] and NLL+NLO results.

one-loop soft anomalous dimension of ref. [38]). We note, however, that the regular component $d \sigma^{(\text {reg) }}$ (see eq. (2.2)) of the NLL+NLO calculation is left unchanged, so as not to spoil the matching procedure with the complete NLO result at low values of $q_{T}$ (removing the effect of $\boldsymbol{\Gamma}_{t}^{(1)}$ from $d \sigma^{(\mathrm{reg})} / d q_{T}$ would lead to a divergent cross section in the limit $q_{T} \rightarrow 0$ ).

The modified (by setting $\boldsymbol{\Gamma}_{t}^{(1)}=0$ in $g_{N}^{(2)}$ ) NLL+NLO result for $d \sigma / d q_{T}$ at central values of the scales $\left(\mu_{F}=\mu_{R}=Q=m_{t}\right)$ is presented in figure 8 (dashed lines), where it is compared to the full NLL+NLO result with its scale dependence (solid lines). Since our modification only affects the function $g_{N}^{(2)}$ in the resummed form factor $\mathcal{G}$, the integral over $q_{T}$ of the dashed histogram in figure 8 coincides with the NLO total cross section. Therefore, at central values of the scales, the solid and dashed histograms in figure 8 differ only in their shape, and the $q_{T}$ shape of solid histogram is softer than that of the dashed histogram. As we can see, the effect of the soft wide-angle radiation (which is included in the full NLL+NLO result) is positive at small values of $q_{T}$ : in the lowest- $q_{T}$ bin, its size is at the level of about $+40 \%$, which is well outside the range of the scale variation band. At larger values of $q_{T}\left(q_{T} \gtrsim 50 \mathrm{GeV}\right)$, the effect is negative and it reaches the size of about $-40 \%$ at $q_{T} \sim 200 \mathrm{GeV}$.

We can present a qualitative interpretation of the results in figure 8. The soft anomalous dimension $\boldsymbol{\Gamma}_{t}^{(1)}$ formally parametrizes the differences at the resummed level between heavy-quark production and the production of a colourless system from the same colliding partons. The term $\boldsymbol{\Gamma}_{t}^{(1)}$ is due to soft radiation at large angles with respect to the direction of the initial-state colliding partons (hadrons). Soft wide-angle radiation receives 


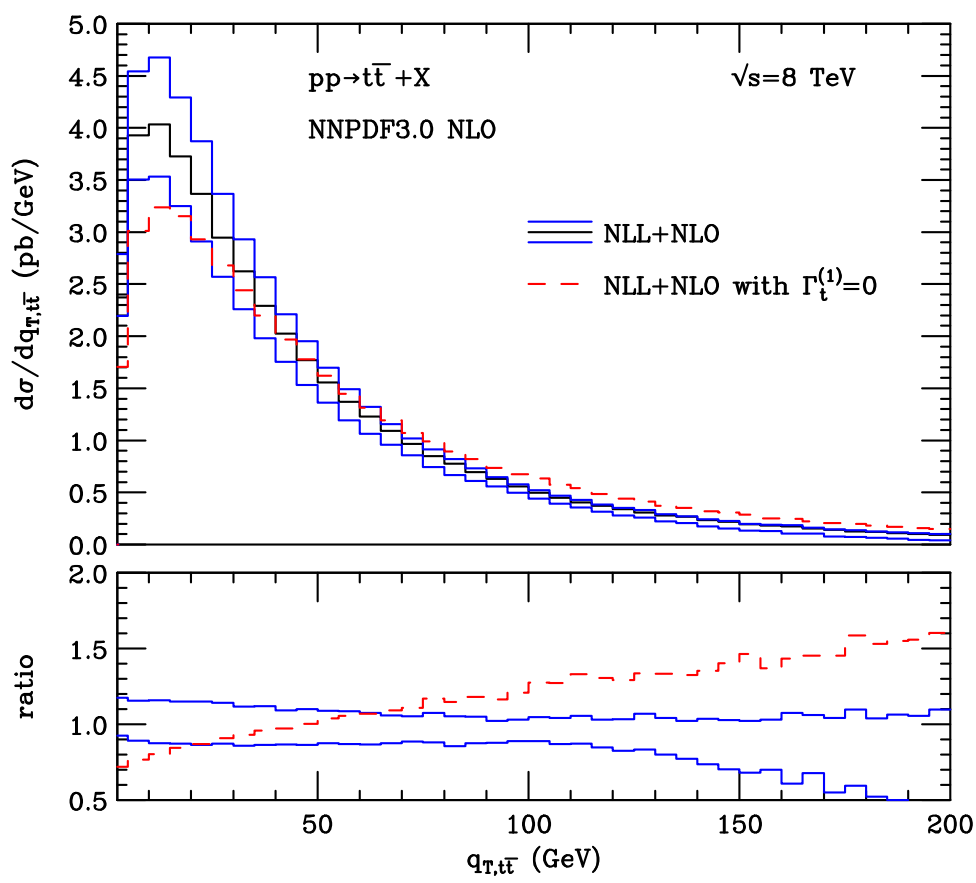

Figure 8. The transverse-momentum cross section $d \sigma / d q_{T}$ of the $t \bar{t}$ pair at the $\operatorname{LHC}(\sqrt{s}=8 \mathrm{TeV})$. The scale dependent result at NLL+NLO accuracy (solid) is compared with the 'modified' result (dashed) that is obtained by removing the resummed contribution of soft wide-angle radiation (which is given by the soft anomalous dimension $\left.\Gamma_{t}^{(1)}\right)$ at central scales $\left(\mu_{R}=\mu_{F}=Q=m_{t}\right)$. The lower panel presents the ratios of the NLL+NLO scale dependence and of the 'modified' result with respect to the NLL+NLO result at central scales.

two physically distinct contributions: final-state radiation from the heavy-quark pair and initial/final-state interferences due to the non-vanishing colour charge of the heavy quarks. Final-state radiation tends to make the $q_{T}$ spectrum harder. Owing to colour-coherence effects, interferences have instead a destructive character and they soften the $q_{T}$ spectrum (a related discussion of colour-coherence in the context of threshold resummation is presented in section 4 of ref. [67]). In the case of $t \bar{t}$ production the colour-coherence destructive interferences dominate with respect to the final-state radiation effect, since the latter is suppressed (screened) by the large value of the top-quark mass. It follows that the presence of $\boldsymbol{\Gamma}_{t}^{(1)}$ leads to a softer $q_{T}$ spectrum in qualitative agreement with the results in figure 8. This is the overall effect of colour-coherence on the $q_{T}$ spectrum. As recalled in section 2 , the colour correlation effects due to $\boldsymbol{\Gamma}_{t}^{(1)}$ have a definite dynamical dependence on the quark (or antiquark) scattering angle $\theta$. Detailed studies of the $q_{T}$ cross section at fixed values of the scattering angle can be useful to futher investigate colour-coherence effects in $t \bar{t}$ production at small values of $q_{T}$.

The resummed predictions that we have presented so far are obtained in a purely perturbative framework. As briefly recalled at the end of section 2, the transverse-momentum distribution of high-mass systems is affected by NP effects, which may become significant as $q_{T} \rightarrow 0$. To check the impact of NP effects at small values of $q_{T}$, we use the customary procedure of multiplying the resummation component of the cross section in $b$ space (see 


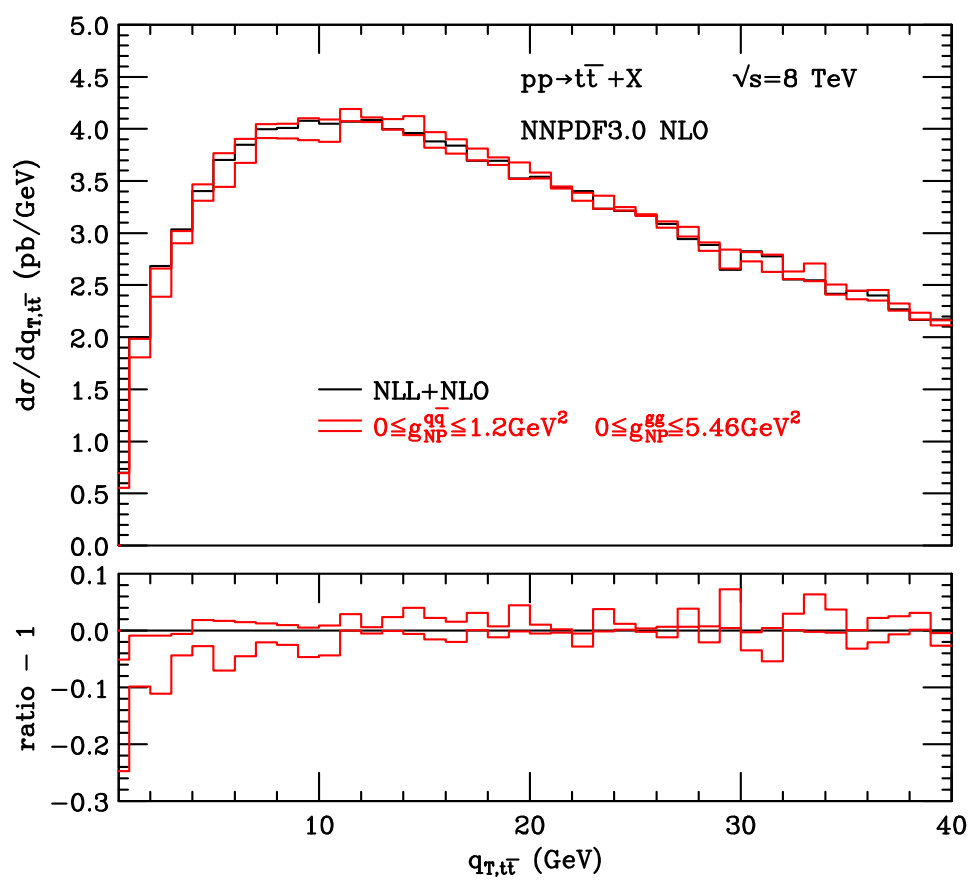

Figure 9. The transverse-momentum cross section $d \sigma / d q_{T}$ of the $t \bar{t}$ pair at the $\operatorname{LHC}(\sqrt{s}=8 \mathrm{TeV})$. Comparison of NLL+NLO results at central scales $\left(\mu_{R}=\mu_{F}=Q=m_{t}\right)$ without (black line) and with (red band) the inclusion of a NP form factor $\left(0 \leq g_{\mathrm{NP}}^{q \bar{q}} \leq 1.2 \mathrm{GeV}^{2}, 0 \leq g_{\mathrm{NP}}^{g g} \leq 5.46 \mathrm{GeV}^{2}\right)$. The lower panel presents the relative effect of the NP form factor.

eq. (2.4)) by a NP smearing form factor $S_{N P}$ of gaussian form:

$$
S_{N P}=\exp \left\{-g_{N P} b^{2}\right\} .
$$

We use different values of the NP parameter $g_{N P}$ for the $q \bar{q}$ annihilation and $g g$ fusion channels (see eq. (2.3)). Specifically, we vary $g_{N P}$ in the range $0 \leq g_{\mathrm{NP}}^{q \bar{q}} \leq 1.2 \mathrm{GeV}^{2}$ for the $q \bar{q}$ driven contribution, and in the range $0 \leq g_{\mathrm{NP}}^{g g} \leq 5.46 \mathrm{GeV}^{2}$ for the $g g$ contribution. These ranges of $g_{N P}$ are those that were considered in ref. [45] and ref. [68] for vector and Higgs boson production, respectively. The quantitative effects of the NP form factor on the NLL+NLO result for $d \sigma / d q_{T}$ are shown in figure 9 . The red band corresponds to the envelope of the NP results that are obtained by varying $g_{N P}^{q \bar{q}}$ and $g_{N P}^{g g}$ in their specified ranges. We see that the NP effects are small and, in particular, definitely smaller than the perturbative uncertanties due to scale variations. This conclusion holds with the exception of the region of very small values of $q_{T}$ (say, $q_{T} \lesssim 5 \mathrm{GeV}$ ), where the impact of NP effects can of the order of $10 \%$. This result is consistent with analogous findings in the case of vector [45] and Higgs [68] boson production.

As stated at the beginning of section 3.2, our resummed results are obtained by using the value $b_{\text {lim }}=3 \mathrm{GeV}^{-1}$ of the parameter $b_{\text {lim }}$ in eq. (2.8). We have examined variations of $b_{\lim }$ by about a factor of two and we find quantitative effects that are definitely smaller than those produced by the NP form factor.

In this paper we do not study how different PDF sets affect the QCD predictions for the $q_{T}$ spectrum of the $t \bar{t}$ pair. A study of the normalized $q_{T}$ distribution $1 / \sigma\left(d \sigma / d q_{T}\right)$ 
at $\sqrt{s}=8 \mathrm{TeV}$ by using different PDF sets is performed by the ATLAS Collaboration in ref. [17]. The results in ref. [17] (see, e.g., figure $6 \mathrm{~b}$ therein) show that various sets of modern PDFs produce differences that are below the $10 \%$ level.

Our NLL+NLO calculation consistently combines the NLO perturbative result, which is well-behaved at high values of $q_{T}$, with the resummation of the logarithmically-enhanced terms in the small- $q_{T}$ region. Using the resummation formalism in impact parameter space (see eq. (2.4)) we achieve control of the large logarithmic terms in analytic form up to NLL accuracy. Parton shower (PS) Monte Carlo event generators provide an alternative way for carrying out the resummation procedure. In this case the resummation of the large logarithmic terms is effectively achieved through the PS, although within the limited logarithmic accuracy of present PS algorithms. The matching of the PS with the exact NLO calculation, which leads to NLO+PS generators, can be carried out by using the MC@NLO [69, 70] or POWHEG [71, 72] methods. Both methods have been applied to $t \bar{t}$ hadroproduction $[70,73]$.

It is of interest to present an illustrative comparison between our NLL+NLO results and those of a NLO+PS Monte Carlo generator. We choose a Monte Carlo generator based on the POWHEG method, and we use the POWHEG BOX implementation [42] to interface the $t \bar{t}$ NLO calculation to the version 6.4.25 of the PYTHIA parton shower [41] with the default values of the parameters. The POWHEG+PYTHIA calculation is performed by varying the parameter $h_{\text {damp }}$ [74]. Within the POWHEG method, the radiation of the final-state parton with the largest transverse-momentum $p_{T}$ ('the hardest radiation') is generated at first (i.e., before parton showering effects) according to the NLO result and it is weighted by a Sudakov form factor that is obtained by exponentiating a fraction $F$ of the NLO real-emission contribution. The fraction $F$ is controlled by the parameter $h_{\text {damp }}$ in the form

$$
F\left(p_{T}\right)=\frac{h_{\text {damp }}^{2}}{h_{\text {damp }}^{2}+p_{T}^{2}},
$$

so that larger values of $h_{\text {damp }}$ are expected to produce a harder $q_{T}$ spectrum of the $t \bar{t}$ pair.

The comparison between our resummed results and those obtained by using POWHEG + PYTHIA is presented in figure 10. We consider the differential cross section $d \sigma / d q_{t}$ for $t \bar{t}$ production at the $\mathrm{LHC}(\sqrt{s}=8 \mathrm{TeV})$, and in this figure we present the corresponding fractional difference (X-'theory')/'theory'. The reference theoretical result ('theory') is the NLL+NLO calculation at central values of the scales $\left(\mu_{R}=\mu_{F}=Q=m_{t}\right)$, and $\mathrm{X}$ denotes either the NLL+NLO scale dependence (computed as in figures 6 and 8 ) or the POWHEG + PYTHIA result at three different values of $h_{\text {damp }}\left(h_{\text {damp }}=\left\{m_{t} / 2, m_{t}, 2 m_{t}\right\}\right)$ and fixed $\mu_{R}=\mu_{F}=m_{t}$. The comparison in figure 10 shows that the NLL+NLO result and the POWHEG+PYTHIA result are consistent with each other within their uncertainties, and they are quite similar at small and intermediate values of $q_{T}$.

We note that the $h_{\text {damp }}$ dependence computed in figure 10 certainly corresponds to a lower limit on the uncertainty of POWHEG+PYTHIA. Indeed, there are various other sources of theoretical uncertainties in NLO+PS event generators (see, e.g., ref. [75]). These include effects from variations of $\mu_{R}$ and $\mu_{F}$, from the detailed matching procedure with the NLO calculation and from the uncertainties of the PS algorithms. 


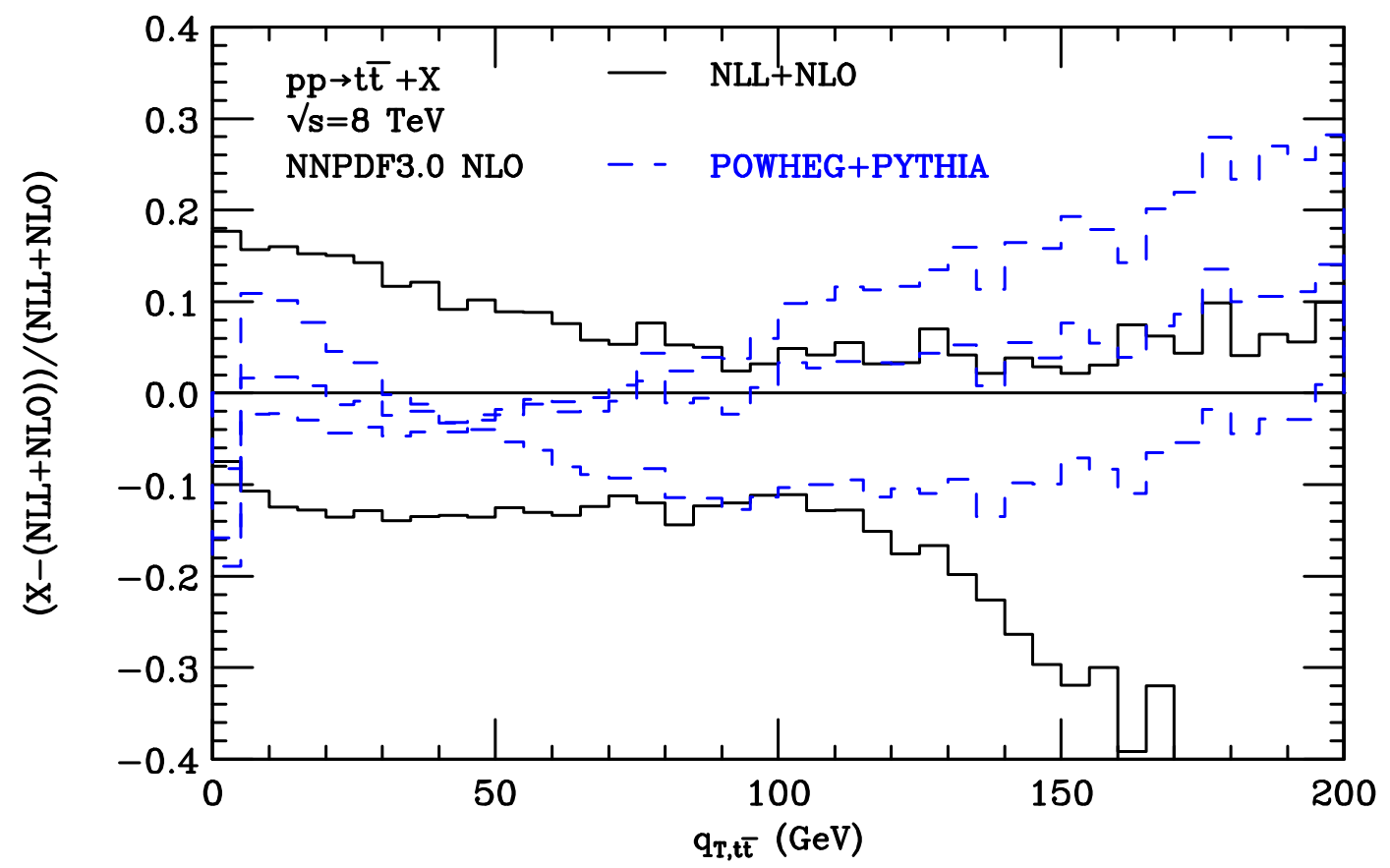

Figure 10. Fractional difference of the POWHEG+PYTHIA results (dashed) with respect to the NLL+NLO result at central scales $\left(\mu_{F}=\mu_{R}=Q=m_{t}\right)$. The results refer to the differential cross section $d \sigma / d q_{T}$ of $t \bar{t}$ pairs at $\sqrt{s}=8 \mathrm{TeV}$. The POWHEG+PYTHIA results are presented for three different values of $h_{\text {damp }}, h_{\text {damp }}=\left\{m_{t} / 2, m_{t}, 2 m_{t}\right\}$, at fixed $\mu_{F}=\mu_{R}=m_{t}$. The scale dependence of the NLL+NLO results (solid) is also reported.

In refs. [17] and [18] ATLAS and CMS data on the $q_{T}$ spectrum of $t \bar{t}$ pairs at the LHC $(\sqrt{s}=8 \mathrm{TeV})$ have been compared with the results of various NLO+PS event generators, which use both the MC@NLO and POWHEG methods interfaced to PYTHIA and HERWIG [76] for parton showering. The comparison shows that the measured $q_{T}$ spectrum is well described $[17,18]$ by these Monte Carlo event generators. Owing to the agreement between these data and our NLL+NLO results (as previously discussed), it turns out that the NLL+NLO results and those of $\mathrm{NLO}+\mathrm{PS}$ generators are relatively similar at small and intermediate values of $q_{T}$.

This similarity is partly expected. The PS algorithm effectively resums LL contributions in the small- $q_{T}$ region. Some contributions at the NLL level are also included through the matching with the NLO result and through the PS. In particular, the PS produces finalstate radiation from the $t \bar{t}$ pair and it can take into account soft-gluon colour-coherence effects (though they are usually approximated in the large- $N_{c}$ limit, $N_{c}$ being the number of colours) [77] by using angular-ordered evolution or dipole showering.

As recalled in section 1, quantitative results of a resummed calculation for the $q_{T}$ spectrum of $t \bar{t}$ pairs at LHC energies were obtained in refs. [36, 37]. The resummed calculation of ref. [37] and our calculation at NLL+NLO accuracy are based on equivalent formal inputs [37, 38], but they use different implementation formalisms. The differences regard, for instance, the type of auxiliary scales that are used to organize the resummed 
calculation, a partly different classification of the resummed logarithmic terms and the inclusion in ref. [37] of some beyond-NLL effects due to the soft anomalous dimension $\boldsymbol{\Gamma}_{t}^{(2)}$ at $\mathcal{O}\left(\alpha_{\mathrm{S}}^{2}\right)[37,38]$. In view of these implementation differences, ensuing quantitative differences between our results and those of ref. [37] are expected. The results of ref. [37] were compared to ATLAS and CMS data in refs. [17, 18]. From that comparison and, in particular, from the Theory/Data plots in figure 9 of ref. [17] and figures 11 and 14 of ref. [18] we can infer an indirect comparison with our results. We find that the $q_{T}$ spectrum of ref. [37] tends to be softer than our spectrum, and still softer than the spectrum of the ATLAS and CMS data. More precisely, in the region of small and medium values of $q_{T}$ (where, say, $q_{T} \lesssim 120 \mathrm{GeV}$ ), the central prediction of ref. [37] is consistent with our results within our scale uncertainty band. At larger values of $q_{T}$, the central prediction of ref. [37] lies outside our scale uncertainty band, and it sizeably deviates from both the NLO result and the data (as pointed out in refs. [17, 18]).

\section{Summary}

In this paper we have considered the transverse-momentum cross section of $t \bar{t}$ pairs at the LHC.

We have first presented fixed-order predictions at NLO (i.e., up to $\left.\mathcal{O}\left(\alpha_{\mathrm{S}}^{3}\right)\right)$ and NNLO (i.e., up to $\mathcal{O}\left(\alpha_{\mathrm{S}}^{4}\right)$ ) and we have compared them with ATLAS and CMS data at $\sqrt{s}=8 \mathrm{TeV}$. At intermediate and large values of $q_{T}$ the NNLO corrections improve the agreement with the data and the NNLO uncertainties are comparable to the experimental uncertainties.

At small values of $q_{T}$ the reliability of the fixed-order perturbative expansion is spoiled by the presence of large logarithmic contributions. These logarithmic contributions have to be resummed to all perturbative orders. Using the resummation formalism of ref. [38] we have presented quantitative results up to NLL+NLO accuracy. The NLL+NLO result has uniform theoretical accuracy throughout the regions of small and intermediate values of $q_{T}$, leading to a perturbative uncertainty of the order of $\pm 10 \%$ for the normalised $q_{T}$ distribution. In these regions the resummed result is consistent with the ATLAS and CMS data at $\sqrt{s}=8 \mathrm{TeV}$ within the corresponding uncertainties. At large values of $q_{T}$ the resummation procedure cannot improve the predictivity of the fixed-order expansion. The NNLO resummation contribution $\mathcal{H}^{(2)}$ (see eq. (2.7)) is not yet known. Once $\mathcal{H}^{(2)}$ becomes available, theoretical improvements of the resummed predictions can be obtained by extending the resummed calculation to NNLL+NNLO accuracy.

The NLL+NLO result for the $q_{T}$ spectrum receives resummed contributions from soft wide-angle radiation through a corresponding soft anomalous dimension. We have examined the quantitative impact of these contributions and we have shown that they make the $q_{T}$ spectrum softer, in qualitative agreement with a discussion of colour-coherence effects. We have finally presented a comparison of our resummed calculation with the prediction of the Monte Carlo event generator POWHEG+PYTHIA. We find that the two predictions are consistent within their estimated uncertainties. 


\section{Acknowledgments}

We are grateful to Stefan Kallweit for his help with the Munich code. This research was supported in part by the Swiss National Science Foundation (SNF) under contract 200020169041 and by the Research Executive Agency (REA) of the European Union under the Grant Agreement number PITN-GA-2012-316704 (Higgstools).

Open Access. This article is distributed under the terms of the Creative Commons Attribution License (CC-BY 4.0), which permits any use, distribution and reproduction in any medium, provided the original author(s) and source are credited.

\section{References}

[1] Particle Data Group collaboration, C. Patrignani et al., Review of particle physics, Chin. Phys. C 40 (2016) 100001 [InSPIRE].

[2] P. Nason, S. Dawson and R.K. Ellis, The total cross-section for the production of heavy quarks in hadronic collisions, Nucl. Phys. B 303 (1988) 607 [INSPIRE].

[3] W. Beenakker, H. Kuijf, W.L. van Neerven and J. Smith, QCD corrections to heavy quark production in $p \bar{p}$ collisions, Phys. Rev. D 40 (1989) 54 [INSPIRE].

[4] W. Beenakker, W.L. van Neerven, R. Meng, G.A. Schuler and J. Smith, QCD corrections to heavy quark production in hadron hadron collisions, Nucl. Phys. B 351 (1991) 507 [INSPIRE].

[5] P. Nason, S. Dawson and R.K. Ellis, The one particle inclusive differential cross-section for heavy quark production in hadronic collisions, Nucl. Phys. B 327 (1989) 49 [Erratum ibid. B 335 (1990) 260] [INSPIRE].

[6] M.L. Mangano, P. Nason and G. Ridolfi, Heavy quark correlations in hadron collisions at next-to-leading order, Nucl. Phys. B 373 (1992) 295 [INSPIRE].

[7] P. Bärnreuther, M. Czakon and A. Mitov, Percent level precision physics at the Tevatron: first genuine NNLO QCD corrections to $q \bar{q} \rightarrow t \bar{t}+X$, Phys. Rev. Lett. 109 (2012) 132001 [arXiv: 1204.5201] [INSPIRE].

[8] M. Czakon and A. Mitov, NNLO corrections to top-pair production at hadron colliders: the all-fermionic scattering channels, JHEP 12 (2012) 054 [arXiv: 1207.0236] [INSPIRE].

[9] M. Czakon and A. Mitov, NNLO corrections to top pair production at hadron colliders: the quark-gluon reaction, JHEP 01 (2013) 080 [arXiv: 1210.6832] [INSPIRE].

[10] M. Czakon, P. Fiedler and A. Mitov, Total top-quark pair-production cross section at hadron colliders through $O\left(\alpha_{S}^{4}\right)$, Phys. Rev. Lett. 110 (2013) 252004 [arXiv: 1303.6254] [INSPIRE].

[11] M. Czakon, P. Fiedler and A. Mitov, Resolving the Tevatron top quark forward-backward asymmetry puzzle: fully differential next-to-next-to-leading-order calculation, Phys. Rev. Lett. 115 (2015) 052001 [arXiv: 1411.3007] [INSPIRE].

[12] M. Czakon, P. Fiedler, D. Heymes and A. Mitov, NNLO QCD predictions for fully-differential top-quark pair production at the Tevatron, JHEP 05 (2016) 034 [arXiv: 1601.05375] [INSPIRE].

[13] M. Czakon, D. Heymes and A. Mitov, Dynamical scales for multi-TeV top-pair production at the LHC, JHEP 04 (2017) 071 [arXiv: 1606. 03350] [INSPIRE]. 
[14] M. Czakon, D. Heymes and A. Mitov, FastNLO tables for NNLO top-quark pair differential distributions, arXiv:1704.08551 [INSPIRE].

[15] CMS collaboration, Measurement of differential top-quark pair production cross sections in pp colisions at $\sqrt{s}=7 \mathrm{TeV}$, Eur. Phys. J. C 73 (2013) 2339 [arXiv:1211.2220] [InSPIRE].

[16] ATLAS collaboration, Differential top-antitop cross-section measurements as a function of observables constructed from final-state particles using pp collisions at $\sqrt{s}=7 \mathrm{TeV}$ in the ATLAS detector, JHEP 06 (2015) 100 [arXiv:1502.05923] [INSPIRE].

[17] ATLAS collaboration, Measurement of top quark pair differential cross-sections in the dilepton channel in pp collisions at $\sqrt{s}=7$ and $8 \mathrm{TeV}$ with ATLAS,

Phys. Rev. D 94 (2016) 092003 [arXiv: 1607.07281] [INSPIRE].

[18] CMS collaboration, Measurement of the differential cross section for top quark pair production in pp collisions at $\sqrt{s}=8 \mathrm{TeV}$, Eur. Phys. J. C 75 (2015) 542 [arXiv: 1505.04480] [INSPIRE].

[19] ATLAS collaboration, Measurements of top-quark pair differential cross-sections in the lepton+jets channel in pp collisions at $\sqrt{s}=8$ TeV using the ATLAS detector,

Eur. Phys. J. C 76 (2016) 538 [arXiv:1511.04716] [InSPIRE].

[20] CMS collaboration, Measurement of double-differential cross sections for top quark pair production in pp collisions at $\sqrt{s}=8 \mathrm{TeV}$ and impact on parton distribution functions, Eur. Phys. J. C 77 (2017) 459 [arXiv:1703.01630] [INSPIRE].

[21] CMS collaboration, Measurement of differential cross sections for top quark pair production using the lepton+jets final state in proton-proton collisions at $13 \mathrm{TeV}$,

Phys. Rev. D 95 (2017) 092001 [arXiv: 1610.04191] [INSPIRE].

[22] ATLAS collaboration, Measurements of top-quark pair differential cross-sections in the e $\mu$ channel in pp collisions at $\sqrt{s}=13 \mathrm{TeV}$ using the ATLAS detector,

Eur. Phys. J. C 77 (2017) 292 [arXiv:1612.05220] [InSPIRE].

[23] CMS collaboration, Measurement of normalized differential $t \bar{t}$ cross sections in the dilepton channel from pp collisions at $\sqrt{s}=13 \mathrm{TeV}$, JHEP 04 (2018) 060 [arXiv:1708.07638] [INSPIRE].

[24] CMS collaboration, Measurement of differential cross sections for the production of top quark pairs and of additional jets in lepton+jets events from pp collisions at $\sqrt{s}=13 \mathrm{TeV}$, Phys. Rev. D 97 (2018) 112003 [arXiv:1803.08856] [INSPIRE].

[25] S. Dittmaier, P. Uwer and S. Weinzierl, NLO QCD corrections to $t \bar{t}+$ jet production at hadron colliders, Phys. Rev. Lett. 98 (2007) 262002 [hep-ph/0703120] [INSPIRE].

[26] S. Dittmaier, P. Uwer and S. Weinzierl, Hadronic top-quark pair production in association with a hard jet at next-to-leading order QCD: phenomenological studies for the Tevatron and the LHC, Eur. Phys. J. C 59 (2009) 625 [arXiv:0810.0452] [inSPIRE].

[27] K. Melnikov and M. Schulze, NLO QCD corrections to top quark pair production in association with one hard jet at hadron colliders, Nucl. Phys. B 840 (2010) 129 [arXiv: 1004.3284] [INSPIRE].

[28] K. Melnikov, A. Scharf and M. Schulze, Top quark pair production in association with a jet: QCD corrections and jet radiation in top quark decays, Phys. Rev. D 85 (2012) 054002 [arXiv:1111.4991] [INSPIRE]. 
[29] Y.L. Dokshitzer, D. Diakonov and S.I. Troian, On the transverse momentum distribution of massive lepton pairs, Phys. Lett. B 79 (1978) 269 [InSPIRE].

[30] Y.L. Dokshitzer, D. Diakonov and S.I. Troian, Hard processes in quantum chromodynamics, Phys. Rept. 58 (1980) 269 [INSPIRE].

[31] G. Parisi and R. Petronzio, Small transverse momentum distributions in hard processes, Nucl. Phys. B 154 (1979) 427 [inSPIRE].

[32] G. Curci, M. Greco and Y. Srivastava, QCD jets from coherent states, Nucl. Phys. B 159 (1979) 451 [inSPIRE].

[33] J.C. Collins, D.E. Soper and G.F. Sterman, Transverse momentum distribution in Drell-Yan pair and $W$ and $Z$ boson production, Nucl. Phys. B 250 (1985) 199 [INSPIRE].

[34] E.L. Berger and R.-B. Meng, Transverse momentum distributions for heavy quark pairs, Phys. Rev. D 49 (1994) 3248 [hep-ph/9310341] [INSPIRE].

[35] S. Mrenna and C.P. Yuan, Effects of QCD resummation on distributions of top-anti-top quark pairs produced at the Tevatron, Phys. Rev. D 55 (1997) 120 [hep-ph/9606363] [INSPIRE].

[36] H.X. Zhu, C.S. Li, H.T. Li, D.Y. Shao and L.L. Yang, Transverse-momentum resummation for top-quark pairs at hadron colliders, Phys. Rev. Lett. 110 (2013) 082001 [arXiv: 1208.5774] [INSPIRE].

[37] H.T. Li, C.S. Li, D.Y. Shao, L.L. Yang and H.X. Zhu, Top quark pair production at small transverse momentum in hadronic collisions, Phys. Rev. D 88 (2013) 074004 [arXiv: 1307.2464] [INSPIRE].

[38] S. Catani, M. Grazzini and A. Torre, Transverse-momentum resummation for heavy-quark hadroproduction, Nucl. Phys. B 890 (2014) 518 [arXiv: 1408.4564] [INSPIRE].

[39] T. Becher, M. Neubert and D. Wilhelm, Electroweak gauge-boson production at small $q_{T}$ : infrared safety from the collinear anomaly, JHEP 02 (2012) 124 [arXiv:1109.6027] [INSPIRE].

[40] G. Bozzi, S. Catani, D. de Florian and M. Grazzini, Transverse-momentum resummation and the spectrum of the Higgs boson at the LHC, Nucl. Phys. B 737 (2006) 73 [hep-ph/0508068] [INSPIRE].

[41] T. Sjöstrand, S. Mrenna and P.Z. Skands, PYTHIA 6.4 physics and manual, JHEP 05 (2006) 026 [hep-ph/0603175] [INSPIRE].

[42] S. Alioli, P. Nason, C. Oleari and E. Re, A general framework for implementing NLO calculations in shower Monte Carlo programs: the POWHEG BOX, JHEP 06 (2010) 043 [arXiv: 1002.2581] [INSPIRE].

[43] S. Catani, M. Grazzini and H. Sargsyan, Azimuthal asymmetries in QCD hard scattering: infrared safe but divergent, JHEP 06 (2017) 017 [arXiv: 1703.08468] [INSPIRE].

[44] J.C. Collins and D.E. Soper, Angular distribution of dileptons in high-energy hadron collisions, Phys. Rev. D 16 (1977) 2219 [INSPIRE].

[45] S. Catani, D. de Florian, G. Ferrera and M. Grazzini, Vector boson production at hadron colliders: transverse-momentum resummation and leptonic decay, JHEP 12 (2015) 047 [arXiv: 1507.06937] [INSPIRE]. 
[46] G. Bozzi, S. Catani, D. de Florian and M. Grazzini, The $q_{T}$ spectrum of the Higgs boson at the LHC in QCD perturbation theory, Phys. Lett. B 564 (2003) 65 [hep-ph/0302104] [INSPIRE].

[47] N. Kidonakis, G. Oderda and G.F. Sterman, Evolution of color exchange in QCD hard scattering, Nucl. Phys. B 531 (1998) 365 [hep-ph/9803241] [INSPIRE].

[48] J.C. Collins and D.E. Soper, Back-to-back jets: Fourier transform from $b$ to $k_{T}$, Nucl. Phys. B 197 (1982) 446 [InSPIRE].

[49] D. de Florian, G. Ferrera, M. Grazzini and D. Tommasini, Higgs boson production at the LHC: transverse momentum resummation effects in the $H \rightarrow 2 \gamma, H \rightarrow W W \rightarrow \ell \nu \ell \nu$ and $H \rightarrow Z Z \rightarrow 4 \ell$ decay modes, JHEP 06 (2012) 132 [arXiv:1203.6321] [INSPIRE].

[50] R. Bonciani, S. Catani, M. Grazzini, H. Sargsyan and A. Torre, The $q_{T}$ subtraction method for top quark production at hadron colliders, Eur. Phys. J. C 75 (2015) 581 [arXiv: 1508.03585] [INSPIRE].

[51] S. Kallweit, MuNICH is the abbreviation of "MUlti-chaNnel Integrator at Swiss (CH) precision" - an automated parton level NLO generator, in preparation.

[52] M. Grazzini, S. Kallweit and M. Wiesemann, Fully differential NNLO computations with MATRIX, Eur. Phys. J. C 78 (2018) 537 [arXiv:1711.06631] [INSPIRE].

[53] S. Catani and M.H. Seymour, The dipole formalism for the calculation of QCD jet cross-sections at next-to-leading order, Phys. Lett. B 378 (1996) 287 [hep-ph/9602277] [INSPIRE].

[54] S. Catani and M.H. Seymour, A general algorithm for calculating jet cross-sections in NLO QCD, Nucl. Phys. B 485 (1997) 291 [Erratum ibid. B 510 (1998) 503] [hep-ph/9605323] [INSPIRE].

[55] S. Catani, S. Dittmaier, M.H. Seymour and Z. Trócsányi, The dipole formalism for next-to-leading order QCD calculations with massive partons, Nucl. Phys. B 627 (2002) 189 [hep-ph/0201036] [INSPIRE].

[56] F. Cascioli, P. Maierhofer and S. Pozzorini, Scattering amplitudes with open loops, Phys. Rev. Lett. 108 (2012) 111601 [arXiv:1111.5206] [INSPIRE].

[57] A. Denner, S. Dittmaier and L. Hofer, Collier: a fortran-based Complex One-Loop LIbrary in Extended Regularizations, Comput. Phys. Commun. 212 (2017) 220 [arXiv:1604.06792] [INSPIRE].

[58] A. Denner and S. Dittmaier, Reduction of one loop tensor five point integrals, Nucl. Phys. B 658 (2003) 175 [hep-ph/0212259] [INSPIRE].

[59] A. Denner and S. Dittmaier, Reduction schemes for one-loop tensor integrals, Nucl. Phys. B 734 (2006) 62 [hep-ph/0509141] [INSPIRE].

[60] A. Denner and S. Dittmaier, Scalar one-loop 4-point integrals, Nucl. Phys. B 844 (2011) 199 [arXiv: 1005.2076] [INSPIRE].

[61] G. Ossola, C.G. Papadopoulos and R. Pittau, CutTools: a program implementing the OPP reduction method to compute one-loop amplitudes, JHEP 03 (2008) 042 [arXiv:0711.3596] [INSPIRE].

[62] A. van Hameren, OneLOop: for the evaluation of one-loop scalar functions, Comput. Phys. Commun. 182 (2011) 2427 [arXiv: 1007.4716] [InSPIRE]. 
[63] M. Czakon and A. Mitov, Top++: a program for the calculation of the top-pair cross-section at hadron colliders, Comput. Phys. Commun. 185 (2014) 2930 [arXiv:1112.5675] [INSPIRE].

[64] NNPDF collaboration, R.D. Ball et al., Parton distributions for the LHC run II, JHEP 04 (2015) 040 [arXiv: 1410.8849] [INSPIRE].

[65] G. Bozzi, S. Catani, G. Ferrera, D. de Florian and M. Grazzini, Transverse-momentum resummation: a perturbative study of $Z$ production at the Tevatron, Nucl. Phys. B 815 (2009) 174 [arXiv:0812.2862] [INSPIRE].

[66] S. Frixione, M.L. Mangano, P. Nason and G. Ridolfi, Top quark distributions in hadronic collisions, Phys. Lett. B 351 (1995) 555 [hep-ph/9503213] [INSPIRE].

[67] S. Catani, M. Grazzini and A. Torre, Soft-gluon resummation for single-particle inclusive hadroproduction at high transverse momentum, Nucl. Phys. B 874 (2013) 720 [arXiv: 1305.3870] [INSPIRE].

[68] D. de Florian, G. Ferrera, M. Grazzini and D. Tommasini, Transverse-momentum resummation: Higgs boson production at the Tevatron and the LHC, JHEP 11 (2011) 064 [arXiv: 1109.2109] [INSPIRE].

[69] S. Frixione and B.R. Webber, Matching NLO QCD computations and parton shower simulations, JHEP 06 (2002) 029 [hep-ph/0204244] [INSPIRE].

[70] S. Frixione, P. Nason and B.R. Webber, Matching NLO QCD and parton showers in heavy flavor production, JHEP 08 (2003) 007 [hep-ph/0305252] [INSPIRE].

[71] P. Nason, A new method for combining NLO QCD with shower Monte Carlo algorithms, JHEP 11 (2004) 040 [hep-ph/0409146] [INSPIRE].

[72] S. Frixione, P. Nason and C. Oleari, Matching NLO QCD computations with parton shower simulations: the POWHEG method, JHEP 11 (2007) 070 [arXiv: 0709.2092] [INSPIRE].

[73] S. Frixione, P. Nason and G. Ridolfi, A positive-weight next-to-leading-order Monte Carlo for heavy flavour hadroproduction, JHEP 09 (2007) 126 [arXiv:0707.3088] [INSPIRE].

[74] S. Alioli, P. Nason, C. Oleari and E. Re, NLO Higgs boson production via gluon fusion matched with shower in POWHEG, JHEP 04 (2009) 002 [arXiv:0812.0578] [INSPIRE].

[75] P. Nason and B. Webber, Next-to-leading-order event generators, Ann. Rev. Nucl. Part. Sci. 62 (2012) 187 [arXiv:1202.1251] [INSPIRE].

[76] G. Corcella et al., HERWIG 6: an event generator for hadron emission reactions with interfering gluons (including supersymmetric processes), JHEP 01 (2001) 010 [hep-ph/0011363] [INSPIRE].

[77] G. Marchesini and B.R. Webber, Simulation of QCD coherence in heavy quark production and decay, Nucl. Phys. B 330 (1990) 261 [INSPIRE]. 